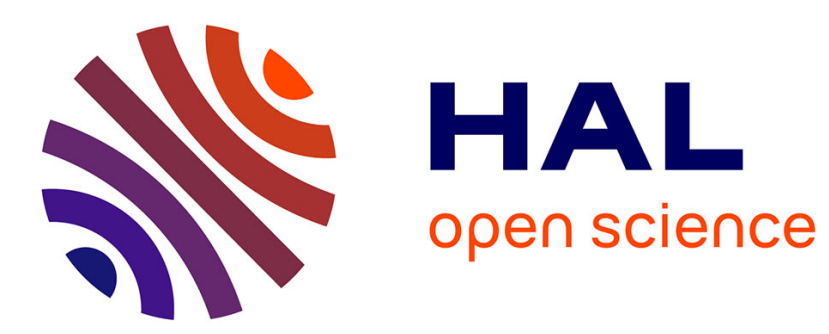

\title{
A model of influence based on aggregation functions
}

Michel Grabisch, Agnieszka Rusinowska

\section{To cite this version:}

Michel Grabisch, Agnieszka Rusinowska. A model of influence based on aggregation functions. 2011. halshs-00639677

\section{HAL Id: halshs-00639677 https://shs.hal.science/halshs-00639677}

Submitted on 9 Nov 2011

HAL is a multi-disciplinary open access archive for the deposit and dissemination of scientific research documents, whether they are published or not. The documents may come from teaching and research institutions in France or abroad, or from public or private research centers.
L'archive ouverte pluridisciplinaire HAL, est destinée au dépôt et à la diffusion de documents scientifiques de niveau recherche, publiés ou non, émanant des établissements d'enseignement et de recherche français ou étrangers, des laboratoires publics ou privés. 


\section{Documents de Travail du Centre d'Economie de la Sorbonne}

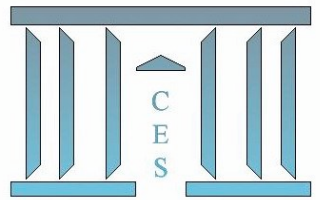

A model of influence based on aggregation functions

Michel GraBisch, Agnieszka RusinOwsKA

2011.58

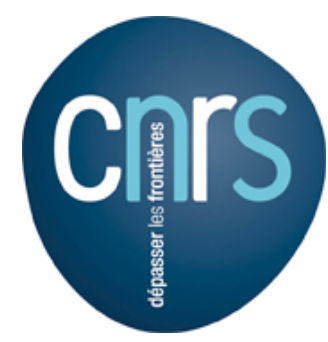




\title{
A model of influence based on aggregation functions*
}

\author{
Michel GRABISCH and Agnieszka RUSINOWSKA \\ Paris School of Economics \\ Université Paris I Panthéon-Sorbonne \\ 106-112 Bd de l'Hôpital, 75647 Paris, France \\ Phone: (+33) 144078 285, Fax: (+33) 144078301 \\ michel.grabisch@univ-paris1.fr, agnieszka.rusinowska@univ-paris1.fr \\ Version of October 7, 2011
}

\begin{abstract}
The paper concerns a dynamic model of influence in which agents have to make a yes-no decision. Each agent has an initial opinion, which he may change during different phases of interaction, due to mutual influence among agents. The influence mechanism is assumed to be stochastic and to follow a Markov chain. In the paper, we investigate a model of influence based on aggregation functions. Each agent modifies his opinion independently of the others, by aggregating the current opinion of all agents, possibly including himself. We provide a general analysis of convergence in the aggregation model and give more practical conditions based on influential players. We show that the process of influence converges always to one of the two consensus states, and there may exist other terminal classes, which are either cyclic or union of Boolean lattices. We give sufficient conditions for avoiding these additional terminal classes, based on properties of the graph of influence and influential players. We also introduce the notion of influential coalition and show that it can fully describe terminal classes. Some important families of aggregation functions are discussed.
\end{abstract}

JEL Classification: C7, D7

Keywords: influence, aggregation function, convergence, terminal class, influential coalition, social network

Corresponding author: Michel Grabisch

\footnotetext{
*We like to thank Philippe Solal and Eric Rémila for pointing out an error in a previous version of Theorem 2. This research project is supported by the National Agency for Research (Agence Nationale de la Recherche), Reference: ANR-09-BLAN-0321-01.
} 


\section{Introduction}

The concepts of interaction and influence in networks are studied in several scientific fields, e.g., in psychology, sociology, economics, mathematics. In the game-theoretical literature, one-step models of influence appeared already more than fifty years ago. For a short survey of cooperative and noncooperative approaches to influence, see, e.g., Grabisch and Rusinowska (2010c). Very important contributions to a study of influence phenomena can be found in the literature on dynamic aspects of influence. An overview of dynamic models of imitation and social influence is provided, e.g., in Jackson (2008); see also Rusinowska (2010) for a general survey of different approaches to influence. The present paper investigates a new approach to influence based on aggregation functions. Before focusing on the model in question, first we briefly survey some selected literature on dynamic models of interaction and influence.

\subsection{Literature on dynamic models of interaction and influence}

One of the leading models of opinion formation has been introduced by DeGroot (1974). In his model, individuals in a society start with initial opinions on a subject. The interaction patterns are described by a stochastic matrix whose entry on row $j$ and column $k$ represents the weight 'that agent $j$ places on the current belief of agent $k$ in forming $j$ 's belief for the next period'. The beliefs are updated over time. Results in Markov chain theory are easily adapted to the model. Several works in the network literature deal with the DeGroot model and its variations. In particular, Jackson (2008) and Golub and Jackson (2010) examine a model, in which agents communicate in a social network and update their beliefs by repeatedly taking weighted averages of their neighbors' opinions. One of the issues in the DeGroot framework that these authors deal with concerns necessary and sufficient conditions for convergence of the social influence matrix and reaching a consensus; see additionally Berger (1981). Jackson (2008) also examines the speed of convergence of beliefs, and Golub and Jackson (2010) analyze in the context of the DeGroot model whether consensus beliefs are "correct", i.e., whether the beliefs converge to the right probability, expectation, etc. The authors consider a sequence of societies, where each society is strongly connected and convergent, and described by its updating matrix. In each social network of the sequence, the belief of each player converges to the consensus limit belief. There is a true state of nature, and the sequence of networks is wise if the consensus limit belief converges in probability to the true state as the size of society grows.

Several other generalizations of the DeGroot model can be found in the literature, e.g., models in which the updating of beliefs can vary in time and circumstances; see e.g. DeMarzo et al. (2003), Krause (2000), Lorenz (2005), Friedkin and Johnsen (1990, 1997). In particular, in the model of information transmission and opinion formation by DeMarzo et al. (2003), the agents in a network try to estimate some unknown parameter, which allows updating to vary over time, i.e., an agent may place more or less weight on his own belief over time. The authors study the case of multidimensional opinions, in which each agent has a vector of beliefs. They show that, in fact, the individuals' opinions can often be well approximated by a one-dimensional line, where an agent's position on the line determines his position on all issues. Friedkin and Johnsen $(1990,1997)$ study 
a similar framework, in which social attitudes depend on the attitudes of neighbors and evolve over time. In their model, agents start with initial attitudes and then mix in some of their neighbors' recent attitudes with their starting attitudes.

The fact that decisions of individuals are often influenced by decisions of other individuals is also stressed by López-Pintado (2008), who studies a network of interacting agents with actions determined by the actions of their neighbors. López-Pintado and Watts (2008) study how social influence determines collective outcomes in a large population of individuals making binary decisions. Influence networks and the role of social influence in determining distinct collective outcomes is also examined in López-Pintado (2010).

Calvó-Armengol and Jackson (2009) consider an overlapping-generations model in which agents, that represent some dynasties forming a community, take binary actions. The state of the community contains the yes-no actions of all dynasties. In each period 'the parent' is randomly selected from the community and replaced by the 'child', and the higher the number of agents that have adopted the yes-action, the greater the propensity of the child to do the same. The overlapping-generations model with the individual decisions generates a Markov process. In particular, the authors relate their framework to a certain model of stochastic recruitment of ants (Kirman (1993)), where the behavior of the ants (also related to some economic examples) is modelled as a Markov process. Kirman et al. (1986) consider an economy with a stochastic communication between agents. Coalitions can form only between linked players, so the admissible coalitions are stochastic, and the economy is represented by a stochastic graph.

There is a numerous literature on social learning, in particular, in the context of social networks; see, e.g., Banerjee (1992), Ellison (1993), Ellison and Fudenberg (1993, 1995), Bala and Goyal (1998, 2001), Gale and Kariv (2003), Celen and Kariv (2004), Banerjee and Fudenberg (2004). In general, in social learning models agents observe choices over time and update their beliefs accordingly, which is different from the model analyzed in our paper, where the choices depend on the influence of others.

Finally, we like to mention the percolation theory (see, e.g., Broadbent and Hammersley (1957), Kesten (1982)) which describes the behavior of connected clusters in a random graph, and the Ising and Potts models in statistical mechanics. The Ising model (Lenz (1920), Ising (1925)) consists of discrete variables called spins that can be in one of two states. The spins are placed on a lattice or graph, where each spin interacts at most with its nearest neighbors. The Potts model (Potts (1952)) is a certain generalization of the Ising model.

\subsection{The present paper}

In the present paper, we extent a framework of influence studied in Grabisch and Rusinowska (2009, 2010a,b, 2011a,b), to a dynamic model of influence based on aggregation functions. In the basic model (Hoede and Bakker (1982)), agents make a yes-no decision on a certain issue, and while each agent has his preliminary opinion (inclination), he may decide differently from that inclination, due to influence between agents. A transformation from the agents' inclinations to their decisions is represented by an influence function. In Grabisch and Rusinowska (2010a), we introduce several tools for analyzing influence in the yes-no model (e.g., influence indices, concepts of follower and kernel), 
and study several examples of influence functions that model typical real-life behaviors. The model is extended to a framework in which every agent has a totally ordered set of possible actions (Grabisch and Rusinowska (2010b)), and to a model with a continuum of actions (Grabisch and Rusinowska (2011b)). In Grabisch and Rusinowska (2009), we show that the framework of influence is more general than a cooperative model of command games presented in Hu and Shapley $(2003 \mathrm{a}, \mathrm{b})$. This line of research is continued in Grabisch and Rusinowska (2011a), where the exact relations between the key concepts of the influence model and the framework of command games are established.

The present paper extends our previous research in several aspects. While influence functions considered so far were deterministic and the framework was a decision process after a single step of influence, we consider now a dynamic influence mechanism which is assumed to be stochastic and to follow a Markov chain. The main contribution of the present paper to the literature on influence is the introduction and study of the influence framework based on aggregation functions. In our model, each agent modifies his opinion independently of the others, by aggregating the current opinion of all agents, possibly including himself. We provide a general analysis of convergence in the aggregation model and give more practical conditions based on influential players. We show that the process of influence converges always to one of the two consensus states, and there may exist other terminal classes, which are either cyclic or union of Boolean lattices. Moreover, we give sufficient conditions for excluding these additional terminal classes, based on properties of the graph of influence and influential players. We also introduce the notion of influential coalition and show that it can fully describe terminal classes.

Despite the existence of numerous studies on influence in social networks, we are not aware of any work in which the aggregation of opinions was different from using a weighted arithmetic mean (convex combination). We briefly discuss related literature on aggregation models in Section 5.

\section{The deterministic and stochastic models of influ- ence}

We consider a set $N:=\{1, \ldots, n\}$ of agents having to make a yes-no decision for approving a bill, a project, a candidate, etc. (typically these agents form a committee). Each agent is supposed to have an initial opinion (called inclination), but during the different phases of the discussion, agents may change their initial opinion due to mutual influence among agents.

We consider first a deterministic model of influence (Grabisch and Rusinowska, 2010a). Supposing that $S$ is the set of agents having inclination 'yes', we denote by $B(S)$ the set of 'yes'-agents after one step of influence. $B: 2^{N} \rightarrow 2^{N}$ is a fixed function, called influence function, which captures all possible situations of influence. It is important to note that to a given situation, depicted by the set of 'yes'-inclined agents, after influence follows always the same outcome (set of agents voting 'yes'). Since the function $B$ is not directly interpretable, many notions have been proposed in Grabisch and Rusinowska (2010a) to 'read' it, as for example the influence index of a coalition upon an agent, follower functions and kernels. We briefly describe the two last ones. 
Given an influence function $B$, the associated follower function $F_{B}: 2^{N} \rightarrow 2^{N}$ assigns to every coalition $S$ the set of its followers, i.e., agents who always follow the opinion ('yes' or 'no') of agents in $S$, provided they are unanimous (see a thorough study of follower functions in Grabisch and Rusinowska (2011a)). Formally,

$$
F_{B}(S)=\left\{i \in N \mid\left[B\left(S^{\prime}\right) \ni i, \forall S^{\prime} \supseteq S\right] \&\left[B\left(S^{\prime}\right) \not \ngtr i, \forall S^{\prime} \subseteq N \backslash S\right]\right\} .
$$

$F_{B}(S)=\emptyset$ means that coalition $S$ has no real influence on the agents. We say that $S$ is a kernel if $F_{B}(S) \neq \emptyset$ but $F_{B}\left(S^{\prime}\right)=\emptyset$ for every $S^{\prime} \subset S$. It means that $S$ is an irreducible group of agents being influential, because if an agent leaves this group, it looses all its followers.

The above notions are too rigid to be of real interest, since it is unlikely that an agent will always react the same way to the opinion of the others. Hence, we are naturally led to consider stochastic influence functions to introduce some flexibility in the model. Given a situation $S$ (set of 'yes'-inclined voters), there is a certain probability $b_{S, T}$ that the set of 'yes'-voters after one step of influence is $T$. If we assume that the process of influence may iterate (several rounds in the discussion), we obtain a stochastic process, depicting the evolution of the coalition of 'yes'-agents along time. We make here the following simplifying assumptions, which seem reasonable in our context of influence:

(i) The process is Markovian, i.e., the probability $b_{S, T}$ depends on $S$ (the present situation) and $T$ (the future situation), and not on the whole history.

(ii) The process is stationary, i.e., $b_{S, T}$ is constant over time.

States of this finite Markovian process are therefore all subsets $S \subseteq N$, representing the set of 'yes'-agents; its transition matrix $\mathbf{B}:=\left[b_{S, T}\right]_{S, T \subseteq N}$ is a $2^{n} \times 2^{n}$ row-stochastic matrix.

The above two assumptions make the analysis of convergence relatively easy, since one can use the classical results of Markov chains theory. We will not detail these results here, since our analysis of convergence, done in the particular case of aggregation models described in the next section, follows another way.

To the transition matrix $\mathbf{B}$ we associate a graph $\Gamma=\left(2^{N}, E\right)$, called the transition graph, where $E$ is the set of arcs. $\Gamma$ is a directed graph (digraph), whose vertices are all possible coalitions, and an arc $(S, T)$ from state $S$ to state $T$ exists if $b_{S, T}>0$. One can consider also valued arcs, where the value of an $\operatorname{arc}(S, T)$ is simply $b_{S, T}$. The transition graph gives a convenient qualitative view of the process, as well as insights on the convergence. To this end, we introduce some terminology. A path in $\Gamma$ from $S$ to $T$ is a sequence of states $S=S_{0}, S_{1}, S_{2}, \ldots, S_{k-1}, S_{k}=T$ such that $\left(S_{i}, S_{i+1}\right) \in E$ for $i=0, \ldots, k-1$. A nonempty collection $\mathcal{C}$ of states is a class if

(i) either $\mathcal{C}=\{S\}$, and there is no state $T$ such that there exists a path from $S$ to $T$ and from $T$ to $S$;

(ii) or for every distinct $S, T \in \mathcal{C}$, there is a path from $S$ to $T$ and from $T$ to $S$, and $\mathcal{C}$ is maximal for this property (i.e., any addition of a new state in $\mathcal{C}$ makes this property false). 
A class is either transient or terminal. It is transient if there are states $S \in \mathcal{C}$ and $T \notin \mathcal{C}$ such that $(S, T) \in E$ (we call it an outgoing arc). Therefore, a terminal class has no outgoing arc. It means that once the process has entered such a class, it will never exit from it. In other words, the process converges always in one of the terminal classes. If a terminal class is reduced to a single state, we call it a terminal state. The aim is therefore to find all terminal classes and terminal states.

We give several examples illustrating the above notions.

Example 1 (The guru influence function). The guru influence function is a deterministic influence function defined as follows. Let $\widetilde{k} \in N$ be a particular agent called the guru, who has the property that every agent always follows the opinion of the guru. Therefore the influence function is given by:

$$
\operatorname{Gur}^{[\widetilde{k}]}(S):=\left\{\begin{array}{ll}
N, & \text { if } \widetilde{k} \in S \\
\emptyset, & \text { if } \widetilde{k} \notin S
\end{array}, \quad \forall S \subseteq N .\right.
$$

For instance, the transition matrix of the guru function for $n=3$ and $\tilde{k}=1$ is

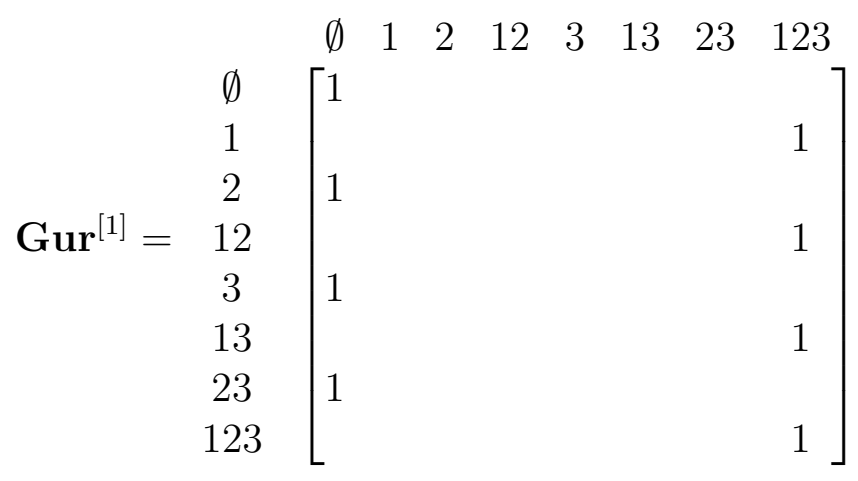

where each "blank" entry means zero, and its associated graph is given in Figure 1. One

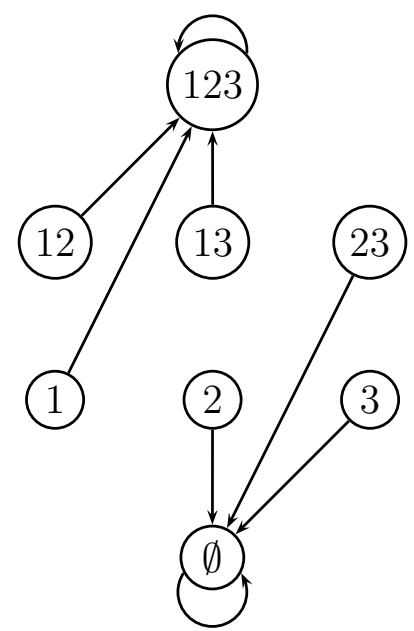

Figure 1: The graph of the guru function Gur ${ }^{[1]}$ for $n=3$

can see that $\emptyset$ and 123 are terminal states. The convergence of the process associated to the guru function is extremely simple: it converges in one step to either $N$ or $\emptyset$, depending whether the opinion of the guru is 'yes' or 'no'. 
Example 2 (The majority influence function). One of the natural ways of making a decision in an influence environment is to decide according to the inclination of the majority. In other words, if the majority of agents has a 'yes' inclination, then all agents decide 'yes', and if not, then all agents decide 'no'. Let $n \geq q>\left\lfloor\frac{n}{2}\right\rfloor$. The majority influence function $\mathrm{Maj}^{[q]}$ is given by

$$
\operatorname{Maj}^{[q]}(S):=\left\{\begin{array}{ll}
N, & \text { if }|S| \geq q \\
\emptyset, & \text { if }|S|<q
\end{array}, \quad \forall S \subseteq N .\right.
$$

Note that this function is also deterministic. The transition graph of the majority influence function for $n=3$ and $q=2$ is given in Figure 2. Obviously, $\emptyset$ and 123 are terminal

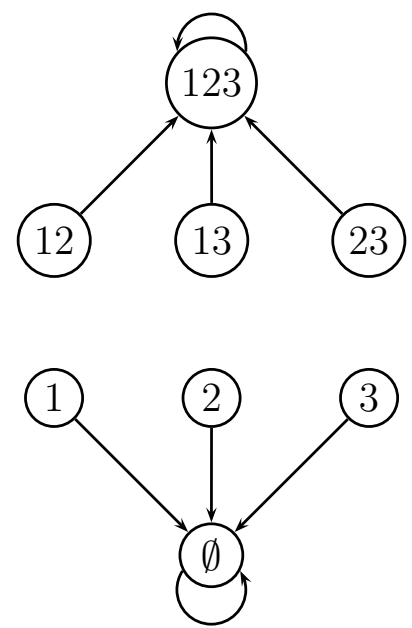

Figure 2: The graph of the majority function $\mathrm{Maj}^{[2]}$ for $n=3$

states. Here also, the convergence is reached in one step.

Example 3 (The stochastic mass psychology function). Let $\varepsilon$ denote either 'yes' or 'no', and $\bar{\varepsilon}$ denote the opposite of $\varepsilon$. Mass psychology influence means that if there is a sufficient number of agents with opinion $\varepsilon$, they will attract some agents with inclination $\bar{\varepsilon}$ and make them change their opinion. Formally, this can be expressed as follows (Grabisch and Rusinowska, 2010a): Let $n \geq q>\left\lfloor\frac{n}{2}\right\rfloor$. The mass psychology function Mass ${ }^{[q]}$ satisfies

$$
\text { if }|S| \geq q \text {, then } \operatorname{Mass}^{[q]}(S) \supseteq S \text {, and if }|N \backslash S| \geq q \text {, then } \operatorname{Mass}^{[q]}(S) \subseteq S \text {, }
$$

where we recall that $S$ is the set of 'yes'-agents.

Here it is natural to consider a stochastic influence function to model the fact that some agents may change their opinion. For instance, the stochastic mass psychology 
function (with uniform distribution) $(n=3, q=2)$ is given by the following matrix:

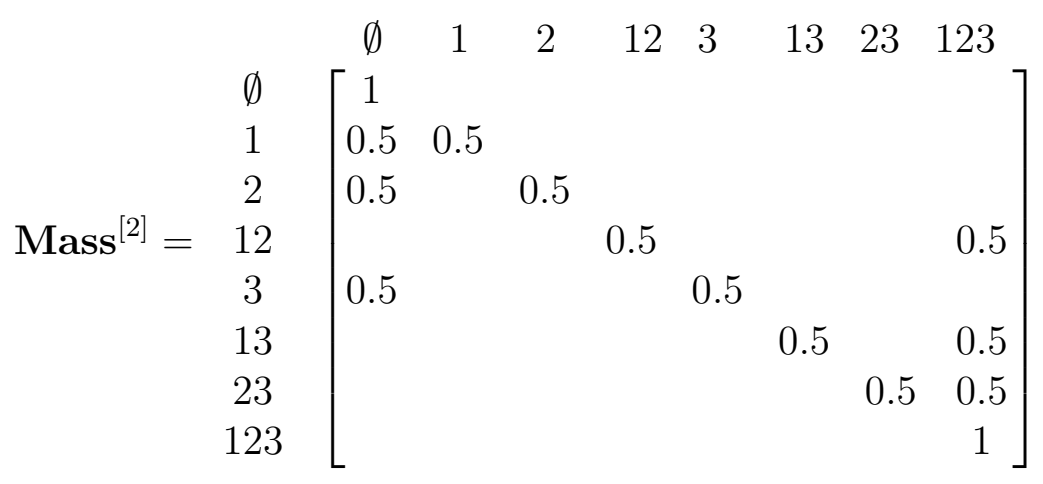

where a "blank" entry means zero. The associated graph is given in Figure 3. Again, $\emptyset$

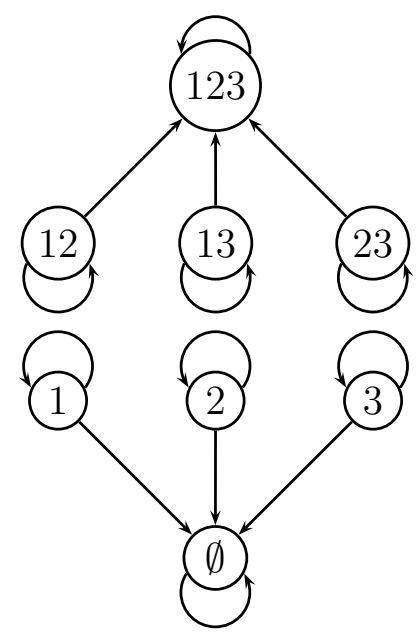

Figure 3: The graph of the stochastic mass psychology function Mass ${ }^{[2]}$ for $n=3$

and 123 are terminal states. For the stochastic mass psychology function in the general case, with $n>3$ and $q>\left\lfloor\frac{n}{2}\right\rfloor$, the following is easy to establish: If the initial state $S(0)$ satisfies $|S(0)| \geq q$, then it converges to $N$ with probability 1 (under some mild conditions on the transition matrix). If $|S(0)| \leq n-q$, then it converges to $\emptyset$ with probability 1 . In all other cases, nothing can be said in general.

\section{Modelling influence by aggregation functions}

The stochastic influence function is a very general model, whose only restrictions are the Markovian and stationarity assumptions. The price to pay for this generality is the exponential complexity of the model: the transition matrix has size $2^{n} \times 2^{n}$, which makes it usable only for a small number of agents.

This motivates the search for subfamilies of polynomial complexity, yet enough general to cover most of real situations. We propose here such a family, whose basic idea is very simple. It assumes that each agent modifies his opinion independently of the other agents, by aggregating the current opinion of all agents, possibly including himself. The precise way of aggregating opinions is characteristic to each agent, so that agents may have all different procedures for aggregating. The aggregation procedure is numerical, coding 'yes' 
by 1 and 'no' by 0 . The result of aggregation is a number between 0 and 1 , representing the probability that the considered agent says 'yes'. For example, the simplest procedure of aggregation is to count the number of 'yes'-agents and to divide by $n$ : the more agents say 'yes', the more you are inclined to say 'yes'. One can also make a weighted count, putting weights on agents ${ }^{1}$, or imagine any kind of procedure, provided it is rational in the following sense: take $S, S^{\prime}$ two sets of 'yes'-agents, and suppose that $S \subseteq S^{\prime}$. Then the probability to say 'yes' for the $S^{\prime}$ situation should be at least equal to the probability for the $S$ situation. This assumption supposes that influence is "positive", that is, agents tends to follow the trend. One can consider as well "negative" influence, where agents modify their opinion in reaction to the opinion of the others: the more agents say 'yes', the more they are inclined to say 'no'. In this case, just the opposite assumption on the aggregation procedure must be taken. In the rest of the paper, we suppose that we deal only with positive influence.

Definition 1. An $n$-place aggregation function is any mapping $A:[0,1]^{n} \rightarrow[0,1]$ satisfying

(i) $A(0, \ldots, 0)=0, A(1, \ldots, 1)=1$ (boundary conditions)

(ii) If $\mathbf{x} \leq \mathbf{x}^{\prime}$ then $A(\mathbf{x}) \leq A\left(\mathbf{x}^{\prime}\right)$ (nondecreasingness).

Aggregation functions are well-studied and there exist many families of them: all kinds of means (geometric, harmonic, quasi-arithmetic) and their weighted version, weighted ordered averages, any combination of minimum and maximum (lattice polynomials or Sugeno integrals), Choquet integrals, triangular norms, copulas, etc. (see Grabisch et al. (2009) and Section 4.6).

To each agent $i \in N$ we associate an aggregation function $A_{i}$, specifying the way agent $i$ modifies his opinion from the opinion of the other agents and himself. Specifically, supposing that $S$ is the set of agents saying 'yes', we compute $\mathbf{x}=\left(A_{1}\left(1_{S}\right), \ldots, A_{n}\left(1_{S}\right)\right)$, where $1_{S}$ is the characteristic vector of $S$, i.e., $\left(1_{S}\right)_{j}=1$ if $j \in S$ and $\left(1_{S}\right)_{j}=0$ otherwise. We denote by $\mathbf{A}:=\left(A_{1}, \ldots, A_{n}\right)$ the vector of aggregation functions, and may use the shorthand $\mathbf{x}=\mathbf{A}\left(1_{S}\right)$.

Vector $\mathbf{x}$ indicates the probability of each agent to say 'yes' after influence. Considering that these probabilities are independent among agents, we find that the probability of transition from the yes-coalition $S$ to the yes-coalition $T$ is

$$
b_{S, T}=\prod_{i \in T} x_{i} \prod_{i \notin T}\left(1-x_{i}\right), \quad \forall S, T \subseteq N,
$$

which determines B. It follows that deterministic models correspond to aggregation functions satisfying $A_{i}\left(1_{S}\right) \in\{0,1\}$ for all $i \in N$, hence they reduce to Boolean functions $B_{i}: 2^{N} \rightarrow\{0,1\}$ which are nondecreasing and nonconstant. Conversely, any deterministic influence model $B: 2^{N} \rightarrow 2^{N}$ satisfying $B(\emptyset)=\emptyset, B(N)=N$ and being nondecreasing is a particular aggregation model, with nondecreasing nonconstant Boolean functions $B_{1}, \ldots, B_{n}$ defined by $B_{i}\left(1_{S}\right)=1$ if $B(S) \ni i$, and 0 otherwise.

Let us show that all our previous examples can be cast into this framework.

\footnotetext{
${ }^{1}$ These weights reflect to which extent an agent takes into account the opinion of the others. For example, in the model of López-Pintado (2010), each agent looks only at the opinion of his neighbors. Note also that if two agents cannot communicate, this can be represented by zero weights.
} 
- The guru and the majority influence functions being deterministic and satisfying the above conditions, it follows that they belong to our aggregation model. For both examples, aggregation functions of all agents are identical. For the guru influence function, we have $A_{i}\left(1_{S}\right)=1$ if $S \ni \vec{k}$ and 0 otherwise, while for the majority influence function we have $A_{i}\left(1_{S}\right)=1$ if $|S| \geq q$, and 0 otherwise.

- Stochastic mass psychology functions can be suitably modelled by aggregation functions. For this, one has to specify for each $S \subseteq N$ the probabilities of transition. If the set $S$ of 'yes' players satisfies $|S|>q$, then there is some probability that players in $N \backslash S$ ('no' players) become 'yes' players (and similarly if $S$ is the set of 'no' players). Suppose that for each situation ( $S \subseteq N, \varepsilon=$ 'yes' or 'no'), the probability $p_{i}^{S, \varepsilon}$ that player $i \in N \backslash S$ changes his opinion is specified, and that players in $N \backslash S$ change independently their opinion. Then this is equivalent to an aggregation model defined as follows, for every $S \subseteq N$ :

$$
A_{i}\left(1_{S}\right)= \begin{cases}1, & \text { if } i \in S \text { and }|S| \geq q \\ p_{i}^{S, y e s}, & \text { if } i \in N \backslash S \text { and }|S| \geq q \\ 0, & \text { if } i \in N \backslash S \text { and }|N \backslash S| \geq q \\ p_{i}^{N \backslash S, \text { no }}, & \text { if } i \in S \text { and }|N \backslash S| \geq q\end{cases}
$$

For example, the transition matrix (1) can be recovered as follows:

$$
\begin{aligned}
& A_{1}\left(\begin{array}{lll}
1 & 0 & 0
\end{array}\right)=0.5 \text {, } \\
& A_{2}\left(\begin{array}{lll}
1 & 0 & 0
\end{array}\right)=0, \\
& A_{3}\left(\begin{array}{lll}
1 & 0 & 0
\end{array}\right)=0 \\
& A_{1}\left(\begin{array}{lll}
0 & 1 & 0
\end{array}\right)=0 \text {, } \\
& A_{2}\left(\begin{array}{lll}
0 & 1 & 0
\end{array}\right)=0.5 \text {, } \\
& A_{3}\left(\begin{array}{lll}
0 & 1 & 0
\end{array}\right)=0 \\
& A_{1}\left(\begin{array}{lll}
0 & 0 & 1
\end{array}\right)=0, \\
& A_{2}\left(\begin{array}{lll}
0 & 0 & 1
\end{array}\right)=0, \\
& A_{3}\left(\begin{array}{lll}
0 & 0 & 1
\end{array}\right)=0.5 \\
& A_{1}\left(\begin{array}{lll}
1 & 1 & 0
\end{array}\right)=1 \text {, } \\
& A_{2}\left(\begin{array}{lll}
1 & 1 & 0
\end{array}\right)=1 \text {, } \\
& A_{3}\left(\begin{array}{lll}
1 & 1 & 0
\end{array}\right)=0.5 \\
& A_{1}\left(\begin{array}{lll}
1 & 0 & 1
\end{array}\right)=1, \\
& A_{2}\left(\begin{array}{lll}
1 & 0 & 1
\end{array}\right)=0.5 \text {, } \\
& A_{3}\left(\begin{array}{lll}
1 & 0 & 1
\end{array}\right)=1 \\
& A_{1}\left(\begin{array}{lll}
0 & 1 & 1
\end{array}\right)=0.5 \text {, } \\
& A_{2}\left(\begin{array}{lll}
0 & 1 & 1
\end{array}\right)=1 \text {, } \\
& A_{3}\left(\begin{array}{lll}
0 & 1 & 1
\end{array}\right)=1 .
\end{aligned}
$$

A natural question is then: when a stochastic influence function does not belong to the class of aggregation models? It suffices that the modification of opinion of agents is correlated: suppose $n=2$, and the two agents are perfectly positively correlated. Then $b_{1, \emptyset}=b_{1,12}=1 / 2, b_{1,1}=b_{1,2}=0$, and similarly for agent 2. From (2), $b_{1,1}=b_{1,2}=0$ implies that $A_{1}\left(1_{1}\right)=A_{2}\left(1_{1}\right)=0$, which makes $b_{1, \emptyset}=b_{1,12}=1 / 2$ impossible.

The following notions will reveal to be central in our analysis.

Definition 2. (i) Let $A_{i}$ be the aggregation function of agent $i$. Agent $j \in N$ is influentiat ${ }^{2}$ in $A_{i}$ if $A_{i}\left(1_{j}\right)>0$ and $A_{i}\left(1_{N \backslash j}\right)<1$.

(ii) The graph of influence is a directed graph $G_{A_{1}, \ldots, A_{n}}=(N, E)$ whose set of nodes is $N$, and there is an arc $(j, i)$ from $j$ to $i$ if $j$ is influential in $A_{i}$. We denote its undirected version by $G_{A_{1}, \ldots, A_{n}}^{0}$.

\footnotetext{
${ }^{2}$ This notion is close to the notion of essential attribute in multiattribute utility theory, see Keeney and Raiffa (1976).
} 
We comment on these two definitions. Roughly speaking, an agent $j$ is influential for agent $i$ if the opinion of $j$ matters for $i$. Indeed, supposing $i \neq j$, even if everybody says 'no' but agent $j$, there is a positive probability that agent $i$ changes his mind due to the influence of $j$. Note that we require also that the same happens when 'no' is replaced by 'yes', since the vector $1_{N \backslash j}$ depicts a situation where every agent says 'yes', except agent $j$. This requirement is natural if we do not want any asymmetry of treatment between 'yes' and 'no'. Note also that the monotonicity of aggregation functions entails that if $j$ is influential in $A_{i}$, then $A_{i}\left(1_{S}\right)>0$ whenever $S \ni j$ and $A_{i}\left(1_{S}\right)<1$ if $S \not \supset j$. Also, if all $A_{i}$ are increasing functions, then the graph of influence is complete with loops.

The graph of influence gives then a clear view of who influences whom. It gives a formal and simple definition of a notion often used in the literature ${ }^{3}$. Figure 4 gives the influential graph of our three examples. The graph of the guru influence function is a
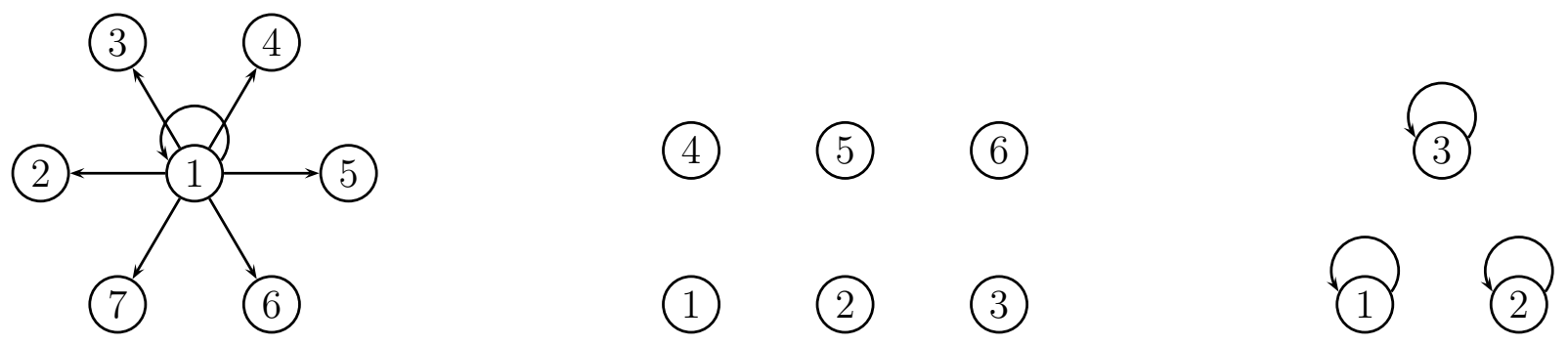

Figure 4: The graph of influence of the guru influence function (left; $n=7$, agent 1 is the guru), the majority influence function (middle, $n=6$ ), and the mass psychology influence function (right, $n=3$, corresponds to the transition matrix (1))

star, showing clearly the rôle of the guru. By contrast, the two other graphs do not reveal anything clear on the influence. This is because no particular agent is really influential in these models. Influence is done only by means of the number of people having the same opinion. We conclude at this stage that graph of influence, though convenient and intuitive, cannot explain all phenomena of influence. In Section 4.5 we introduce other tools able to model this.

\section{Convergence in the aggregation model}

We provide here a general analysis of convergence of influence functions based on aggregation functions.

\subsection{Terminal states}

In all examples given above, we can see that the process converges to the consensus states $N$ or $\emptyset$. We call trivial terminal classes the classes $\{\emptyset\}$ and $\{N\}$. Our first result shows when these are the only terminal states.

\footnotetext{
${ }^{3}$ Aracena et al. (2004) define the connection graph, which is very close to ours: there is an arc $(i, j)$ if the (Boolean) aggregation function $A_{j}$ depends on the input of $i$. Our definition is slightly more restrictive.
} 
Theorem 1. Suppose $\mathbf{B}$ is obtained from an aggregation model, with aggregation functions $A_{1}, \ldots, A_{n}$. Then

(i) The trivial terminal classes are always terminal classes.

(ii) Coalition $S$ is a terminal state ${ }^{4}$ if and only if

$$
A_{i}\left(1_{S}\right)=1 \quad \forall i \in S \text { and } A_{i}\left(1_{S}\right)=0 \text { otherwise. }
$$

(iii) There are no other terminal states than the trivial terminal classes if and only if for all $S \subset N, S \neq \emptyset$, either there is some $i \in S$ such that $A_{i}\left(1_{S}\right)<1$ or there is some $i \in N \backslash S$ such that $A_{i}\left(1_{S}\right)>0$.

(iv) There are no other terminal states than the trivial terminal classes if the undirected graph $G_{A_{1}, \ldots, A_{n}}^{0}$ is connected.

(see proof in the appendix)

We make some comments on this result.

- Unsurprisingly, the consensus states are always terminal states. This is reminiscent of the result of DeGroot for the continuous opinion model.

- Condition (ii) is the basic fact on which the whole theorem is built. In some sense it means that $S$ forms a subsociety opposed to the rest of the society: if $S$ reach the 'yes' consensus, then it remains in this state, and all other agents unanimously say 'no'. Expressing negation of (ii) for every coalition $S$ gives condition (iii). It does not seem possible to find more handy necessary and sufficient conditions.

- Condition (iv) is very easy to verify and intuitively appealing. Indeed, suppose that the graph $G_{A_{1}, \ldots, A_{n}}^{0}$ is not connected. Then there exists a subset of agents who has no relation of influence with the remaining agents. Therefore, this subset of agents may reach a different consensus than the other group.

- Condition (iv) is only a sufficient condition, as shown in Example 4 (ii) below. We will see later under which conditions it becomes also necessary (see Corollary 2).

- As an immediate consequence of condition (iv), it suffices that there exists $i \in N$ such that all agents $j \neq i$ are influential in $A_{i}$. Note that here agent $i$ is the center of a star in the graph of influence with the arcs going into $i$, which means that he takes into account the opinion of all agents.

- The above result says nothing about the existence of terminal classes which are not reduced to singletons. In Example 5, no nontrivial terminal state can exist since condition (iii) is satisfied, however a terminal class exists. The analysis of terminal classes will be done in the next theorem.

Example 4. (i) In the guru example, $G_{A_{1}, \ldots, A_{n}}^{0}$ is a star centered on $\tilde{k}$ with the $\operatorname{arcs}$ going from $\tilde{k}$ into all agents (see Figure 4 ), hence condition (iv) is fulfilled.

\footnotetext{
${ }^{4}$ Note that a terminal state is a fixed point of $\mathbf{A}$.
} 
(ii) In the majority and mass psychology examples, condition (iv) is not fulfilled. Since the only terminal classes are the trivial ones, this shows that (iv) is not a necessary condition.

Example 5. Consider $N=\{1,2,3\}$ and the following aggregation functions:

$$
\begin{array}{ccc}
A_{1}\left(\begin{array}{lll}
1 & 0 & 0
\end{array}\right)=1 & A_{2}\left(\begin{array}{lll}
1 & 0 & 0
\end{array}\right)=0.5 & A_{3}\left(\begin{array}{lll}
1 & 0 & 0
\end{array}\right)=0 \\
A_{1}\left(\begin{array}{lll}
0 & 1 & 0
\end{array}\right)=0 & A_{2}\left(\begin{array}{lll}
0 & 1 & 0
\end{array}\right)=0.5 & A_{3}\left(\begin{array}{lll}
0 & 1 & 0
\end{array}\right)=0 \\
A_{1}\left(\begin{array}{lll}
0 & 0 & 1
\end{array}\right)=0 & A_{2}\left(\begin{array}{lll}
0 & 0 & 1
\end{array}\right)=0.5 & A_{3}\left(\begin{array}{lll}
0 & 0 & 1
\end{array}\right)=0.5 \\
A_{1}\left(\begin{array}{lll}
1 & 1 & 0
\end{array}\right)=1 & A_{2}\left(\begin{array}{lll}
1 & 1 & 0
\end{array}\right)=0.5 & A_{3}\left(\begin{array}{lll}
1 & 1 & 0
\end{array}\right)=0 \\
A_{1}\left(\begin{array}{lll}
1 & 0 & 1
\end{array}\right)=1 & A_{2}\left(\begin{array}{lll}
1 & 0 & 1
\end{array}\right)=0.5 & A_{3}\left(\begin{array}{lll}
1 & 0 & 1
\end{array}\right)=0.5 \\
A_{1}\left(\begin{array}{lll}
0 & 1 & 1
\end{array}\right)=1 & A_{2}\left(\begin{array}{llll}
0 & 1 & 1
\end{array}\right)=0.5 & A_{3}\left(\begin{array}{llll}
0 & 1 & 1
\end{array}\right)=1
\end{array}
$$

This gives the following digraph for the Markov chain:

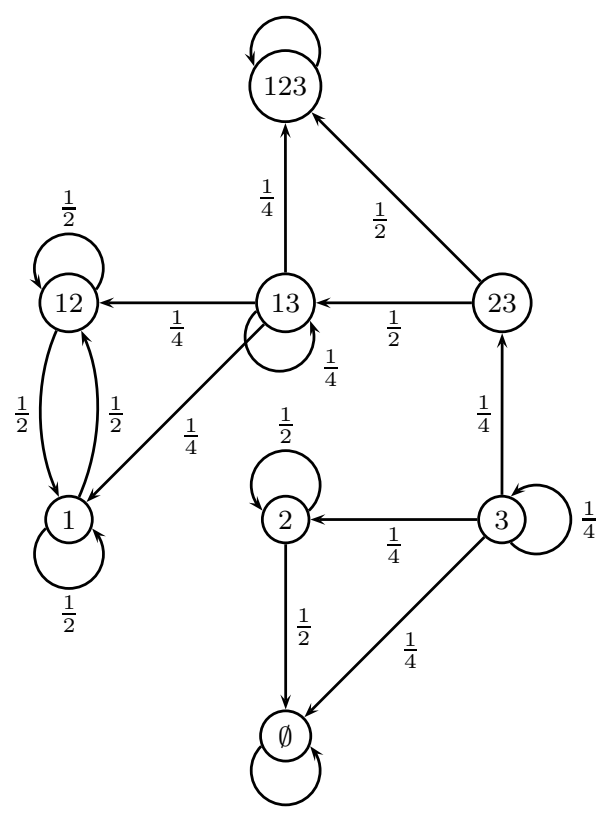

Clearly, $\{1,12\}$ is a terminal class, but not reduced to a singleton. $A_{2}$ has influential players 1 and 3 , so condition (iv) is satisfied.

\subsection{The general result}

We turn to the study of terminal classes not necessarily reduced to a single state.

Theorem 2. Suppose $\mathbf{B}$ is obtained from an aggregation model, with aggregation functions $A_{1}, \ldots, A_{n}$. Then terminal classes are:

(i) either singletons $\{S\}, S \in 2^{N}$,

(ii) or cycles of nonempty sets $\left\{S_{1}, \ldots, S_{k}\right\}$ of any length $2 \leq k \leq\left(\begin{array}{c}n \\ \lfloor n / 2\rfloor\end{array}\right)$ (and therefore they are periodic of period $k$ ) with the condition that all sets are pairwise incomparable (by inclusion) 
(iii) or collections $\mathcal{C}$ of nonempty sets with the property that $\mathcal{C}=\mathcal{C}_{1} \cup \cdots \cup \mathcal{C}_{p}$, where each subcollection $\mathcal{C}_{j}$ is a Boolean lattice $\left[S_{j}, S_{j} \cup K_{j}\right], S_{j} \neq \emptyset, S_{j} \cup K_{j} \neq N$, and at least one $K_{j}$ is nonempty.

We call cyclic terminal classes those terminal classes of the second type and regular terminal classes those of the third type. Regular terminal classes can be periodic (see Example 6 below). Regular terminal classes formed by a single Boolean lattice $[S, S \cup K]$ are called Boolean terminal classes.

(see proof in the appendix)

We give some comments on this result. Example 5 shows the existence of regular terminal classes. The existence of cyclic terminal classes was already remarked by Aracena et al. (2004) for the case of regular aggregation functions, together with the condition of incomparability among sets in the cycle. We know by Sperner's lemma that the longest possible sequence of incomparable sets in $2^{N}$ has length $\left(\begin{array}{c}n \\ \lfloor n / 2\rfloor\end{array}\right)$, hence the upper limit of length. The next proposition is an easy result adapted from Aracena et al. (2004) showing how to construct cyclic classes of maximal length.

Proposition 1. Let $\mathcal{S}:=\left\{S_{0}, S_{1}, \ldots, S_{p-1}\right\}, p \geq 2$, be a sequence of sets, pairwise incomparable, of maximal length. Let $\mathcal{S}^{c}$ be the complementary sequence, i.e., $\mathcal{S}^{c}=$ $2^{N} \backslash \mathcal{S}$, ordered with inclusion (increasingly). Let $A_{1}, \ldots, A_{n}$ be aggregation functions satisfying:

$$
\begin{aligned}
A_{i}\left(1_{S_{j}}\right) & =1, \quad \forall i \in S_{j+1}, \forall j=0, \ldots, p-2 \\
A_{i}\left(1_{S_{j}}\right) & =0, \quad \forall i \notin S_{j+1}, \forall j=0, \ldots, p-2 \\
A_{i}\left(1_{S_{p-1}}\right) & =1, \quad \forall i \in S_{0} \\
A_{i}\left(1_{S_{p-1}}\right) & =0, \quad \forall i \notin S_{0} \\
A_{i}\left(1_{T}\right) & =\left\{\begin{array}{ll}
1, \forall i \in N, & \text { if } \exists S \in \mathcal{S}, S \subset T \\
0, \forall i \in N, & \text { if } \exists S \in \mathcal{S}, S \supset T
\end{array}, \quad \forall T \in \mathcal{S}^{c} .\right.
\end{aligned}
$$

Then $\mathcal{S}$ is a cyclic class of length $p$ of maximal length.

Cyclic classes are not the only case of periodic terminal classes, as the following example shows.

Example 6. Consider $N=\{1,2,3\}$ and the following aggregation functions:

$$
\begin{aligned}
& \mathbf{A}\left(\begin{array}{lll}
1 & 0 & 0
\end{array}\right)=\mathbf{A}\left(\begin{array}{lll}
1 & 1 & 0
\end{array}\right)=\left(\begin{array}{lll}
0 & x & 1
\end{array}\right) \\
& \mathbf{A}\left(\begin{array}{llll}
0 & 0 & 1
\end{array}\right)=\mathbf{A}\left(\begin{array}{lll}
0 & 1 & 1
\end{array}\right)=\left(\begin{array}{lll}
1 & x & 0
\end{array}\right) \\
& \mathbf{A}\left(\begin{array}{llll}
0 & 1 & 0
\end{array}\right)=\mathbf{A}\left(\begin{array}{llll}
0 & 0 & 0
\end{array}\right)=\left(\begin{array}{lll}
0 & 0 & 0
\end{array}\right) \\
& \mathbf{A}\left(\begin{array}{lll}
1 & 0 & 1
\end{array}\right)=\mathbf{A}\left(\begin{array}{lll}
1 & 1 & 1
\end{array}\right)=\left(\begin{array}{lll}
1 & 1 & 1
\end{array}\right)
\end{aligned}
$$

with arbitrary $0<x<1$. Then $\{1,3,12,23\}$ forms a periodic terminal class of period 2 (see Figure 5, left). Now consider the following aggregation functions:

$$
\begin{gathered}
\mathbf{A}\left(\begin{array}{lll}
1 & 0 & 0
\end{array}\right)=\mathbf{A}\left(\begin{array}{lll}
1 & 1 & 0
\end{array}\right)=\left(\begin{array}{lll}
0 & 0 & 1
\end{array}\right) \\
\mathbf{A}\left(\begin{array}{lll}
0 & 0 & 1
\end{array}\right)=\left(\begin{array}{lll}
1 & x & 0
\end{array}\right) \\
\mathbf{A}\left(\begin{array}{lll}
0 & 1 & 0
\end{array}\right)=\mathbf{A}\left(\begin{array}{lll}
0 & 0 & 0
\end{array}\right)=\left(\begin{array}{lll}
0 & 0 & 0
\end{array}\right) \\
\mathbf{A}\left(\begin{array}{lll}
1 & 0 & 1
\end{array}\right)=\mathbf{A}\left(\begin{array}{lll}
0 & 1 & 1
\end{array}\right)=\mathbf{A}\left(\begin{array}{lll}
1 & 1 & 1
\end{array}\right)=\left(\begin{array}{lll}
1 & 1 & 1
\end{array}\right)
\end{gathered}
$$

with arbitrary $0<x<1$. Then $\{1,3,12\}$ is a periodic class of period 2 with 3 sets. 

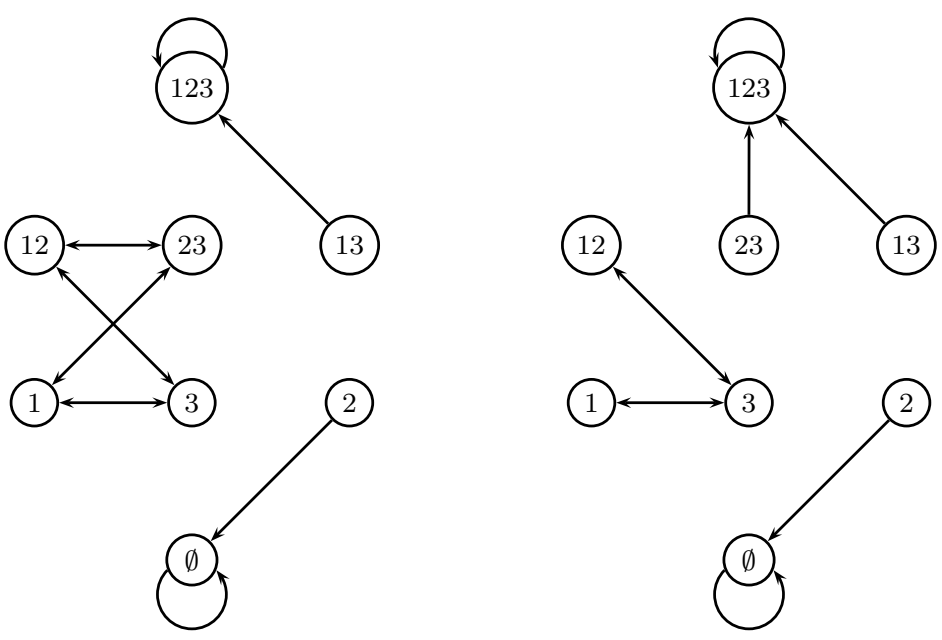

Figure 5: Examples of a periodic terminal class

Generally speaking, to construct a periodic regular terminal class of period $k$, one needs $k$ pairwise disjoint subcollections $\left[S_{j}, S_{j} \cup K_{j}\right], j=1, \ldots, k$, with the usual restrictions on the $S_{j}, K_{j}$ 's, and defines $A_{i}\left(1_{S}\right)=1$ if $\left.i \in S_{j+1}, A_{i}\left(1_{S}\right)=x \in\right] 0,1\left[\right.$ if $i \in K_{j+1}$, and 0 otherwise, for all $S \in\left[S_{j}, S_{j} \cup K_{j}\right]$, identifying $k+1$ with 1 . Note that there is no need to have the $K_{j}$ 's of equal size (see Figure 5 , right).

\subsection{Regular terminal classes}

Let us find some sufficient conditions so that regular terminal classes cannot exist. First we set some notations. A regular terminal class has the form $\mathcal{C}=\bigcup_{k=1}^{p}\left[S_{k}, S_{k} \cup K_{k}\right]$, with the restrictions on $S_{k}, K_{k}$ as in Theorem 2. Let us introduce the lower and upper bounds of $\mathcal{C}$ :

$$
S_{*}=\bigcap_{k=1}^{p} S_{k}, \quad S^{*}=\bigcup_{k=1}^{p}\left(S_{k} \cup K_{k}\right) .
$$

Clearly, $\left[S_{*}, S^{*}\right] \supseteq \mathcal{C}$. Note that $S^{*} \backslash S_{*} \neq \emptyset$ since $\mathcal{C}$ is not a singleton, but $S_{*}=\emptyset, S^{*}=N$ are possible (see Figure 5 , left). If $S_{*} \neq \emptyset$ and $S^{*} \neq N$, we say that the class is normal.

Theorem 3. There is no normal regular terminal class if there is no subgraph $S$ of $G_{A_{1}, \ldots, A_{n}}$ satisfying the following two conditions:

(i) There is no ingoing arc into $S$

(ii) There exists an agent $i \in N \backslash S$ which is not related to $S$, i.e., there is no path from an agent in $S$ to $i$.

(see proof in the appendix)

We make some comments on this result.

- Consider a Boolean terminal class $[S, S \cup K]$. It means that no consensus is reached, but all agents in $S$ agree to say 'yes', while all agents in $N \backslash(S \cup K)$ agree to say 'no'. The agents in $K$ oscillate between 'yes' and 'no' in all possibles ways, without ending. If there are several Boolean lattices in the class, the interpretation is more complex, and depends on how exactly are the transitions between the lattices. 
- The contraposition of Theorem 3 is interesting as well: if there is a normal regular class with upper and lower bounds $S^{*}, S_{*}$, then the subgraph $S_{*}$ has no ingoing arc, and the set of agents which are not related to $S_{*}$ by some path outgoing from $S_{*}$ is exactly $N \backslash S^{*}$ (see proof of Theorem 3). It clearly shows that $S_{*}$ forms an isolated group, receiving no influence, and influencing only the agents in $S^{*} \backslash S_{*}$. Hence, $S^{*}$ forms a subsociety, ruled by $S_{*}$.

- There are two simple particular cases where regular terminal classes cannot occur: when $G_{A_{1}, \ldots, A_{n}}$ is strongly connected, or when there exists one agent who is influential for all agents. $G_{A_{1}, \ldots, A_{n}}$ strongly connected means that more or less directly, all agents are influential for all agents, therefore a dichotomy among agents cannot occur. Also, if one agent is influential for all the others, a consensus will finally emerge around this agent.

- A slight adaptation of the proof permits to write a sufficient condition for regular terminal classes with either $S_{*}=\emptyset$ or $S^{*}=N$ : there is no regular class with $S_{*}=\emptyset, S^{*} \neq N$ (respectively, with $S_{*} \neq \emptyset, S^{*}=N$ ) if there is no subgraph without outgoing arc (respectively, without ingoing arc). However, these conditions are very strong, therefore of little use. Note that there is no way to exclude regular classes with $S_{*}=\emptyset$ and $S^{*}=N$ by imposing some conditions on influential players (this is clear from Lemma 5 in the appendix).

\subsection{Cyclic terminal classes}

We turn to the study of cyclic classes.

Lemma 1. Two simple (but strong) sufficient conditions to forbidding any cyclic terminal class are:

(i) There exists $j \in N$ such that $A_{j}$ takes values 0,1 only for $\emptyset, N$.

(ii) There exists $j \in N$ such that all agents are influential for $A_{j}$.

Proof. (i) Because of $A_{j}$, for no set $S \neq \emptyset, N$, there can be a transition to a set $T$ with probability 1, which forbids the existence of cycles.

(ii) If all agents are influential in $A_{j}$, then $0<A_{j}\left(1_{i}\right)<1$ for all $i \in N$. Indeed, $1=A_{j}\left(1_{i}\right) \leq A_{j}\left(1_{N \backslash k}\right)$ for any $k \neq i$, contradicting the fact that $k$ is influential in $A_{j}$. Similarly, $0<A_{j}\left(1_{N \backslash i}\right)<1$ holds for any $i \in N$. It follows by monotonicity that $A_{j}\left(1_{S}\right) \neq 0,1$ for all $S \neq \emptyset, N$, which by (i) proves the result.

Let us try to refine these conditions. The following lemma is fundamental.

Lemma 2. Suppose $j$ is influential in $A_{i}$. Then there is no cyclic class containing consecutive coalitions $S_{1}, S_{2}$ (in this order) such that $S_{1} \ni j$ and $S_{2} \not \supset i$, or $S_{1} \not \ngtr j$ and $S_{2} \ni i$. (put differently: suppose there is a cyclic class with $S_{1}, S_{2}$ consecutive. Then $A_{i}$ cannot have an influential player $j \in N \backslash S_{1}$ if $i \in S_{2}$, or an influential player $j \in S_{1}$ if $i \notin S_{2}$ ). 
Proof. Since $S_{2}$ is the successor of $S_{1}$ in the cycle, we have $A_{i}\left(1_{S_{1}}\right)=1$ if $i \in S_{2}$ and 0 otherwise. Suppose that $j \in S_{1}$ is influential in $A_{i}$ with $i \notin S_{2}$. Then $0<A_{i}\left(1_{j}\right) \leq$ $A_{i}\left(1_{S_{1}}\right)=0$, a contradiction. Similarly, if $j \notin S_{1}$ is influential in $A_{i}$ with $i \in S_{2}$, we have $1=A_{i}\left(1_{S_{1}}\right) \leq A_{i}\left(1_{N \backslash j}\right)<1$, again a contradiction.

An easy consequence is the following.

Corollary 1. If $i$ is influential in $A_{i}$ for all $i \in N$, there is no cyclic class.

Proof. From Lemma 2, $i$ influential in $A_{i}$ forbids any cycle with consecutive $S_{1}, S_{2}$ such that $S_{1} \ni i$ and $S_{2} \not \ngtr i$, or $S_{1} \not \ngtr i$ and $S_{2} \ni i$. Since $S_{1}, S_{2}$ must be incomparable, $S_{1}=\{i\}$ is ruled out, as well as $S_{1}=N \backslash i$. Take $S_{1} \subset N, S_{1} \neq \emptyset$. Then $S_{2}=L \cup T$, with $L \subseteq N \backslash S_{1}, L \neq \emptyset$ and $T \subseteq S_{1}$. Take $i \in S_{1}$. The fact that $i$ is influential in $A_{i}$ forbids $T \not \ngtr i$, for all $L$. Since this holds for all $i \in S_{1}$, it follows that no $T$ is possible, hence no $S_{2}$.

\subsection{Influential coalitions}

While influential players provide a simple and intuitive way to describe the properties of aggregation functions in terms of influence, they can provide only sufficient conditions, which may become far too restrictive to be of interest. Intuitively speaking, the more agents the less likely a single agent can be influential, which implies that the previous results are of little use in case of a large number of agents. Therefore, we propose a generalization of the notion of influential agent.

Definition 3. Let $A_{i}$ be the aggregation function of agent $i$. A nonempty coalition $S \subseteq N$ is influential in $A_{i}$ if the following two conditions are satisfied:

(i) $A_{i}\left(1_{S}\right)>0$ and $A_{i}\left(1_{N \backslash S}\right)<1$

(ii) For all $S^{\prime} \subset S$, either $A_{i}\left(1_{S^{\prime}}\right)=0$ or $A_{i}\left(1_{N \backslash S^{\prime}}\right)=1$.

We make some comments on this definition.

- The first condition says that the opinion of coalition $S$, when unanimous, matters for agent $i$. Indeed, if agents in $S$ say 'yes' and every other agent says 'no', then agent $i$ has a positive probability to say 'yes'. Similarly, if the set of 'no' voters is $S$, then agent $i$ has a positive probability to say 'no'. The second condition merely says that no subcoalition of $S$ satisfies the first condition, which means that $S$ is truly influential, i.e., it has no superfluous agent.

- The previous definition of an agent influential in $A_{i}$ is clearly a particular case of this definition.

- By the second condition, the set of influential coalitions form an antichain in $2^{N}$ (i.e., they are pairwise incomparable by inclusion).

- The notion of influential coalition is close to and generalizes followers and kernels of the deterministic model of influence (see Section 2). Let $\mathbf{A}$ be Boolean (i.e., the influence function $B$ is deterministic). Suppose that $S$ is influential for $i$. This 
implies $A_{i}\left(1_{S}\right)=1$ and $A_{i}\left(1_{N \backslash S}\right)=0$. By monotonicity, it follows that $A_{i}\left(1_{S^{\prime}}\right)=1$ and $A_{i}\left(1_{N \backslash S^{\prime}}\right)=0$ for any $S^{\prime} \supseteq S$, which is equivalent to say that $i$ is a follower of $S$. The converse is false however, since condition (ii) above may not be satisfied when $i$ is a follower of $S$.

On the other hand, if $S$ is a kernel, then it is influential for some $i$. Observe however that the converse is not true, since it may be the case that if $S$ is influential for $i$, a proper subset $S^{\prime}$ of it may be influential for some $j \neq i$.

Hence a generalization of the follower function for the stochastic case could be:

$$
F_{\mathbf{A}}(S)=\left\{i \in N \mid \exists S^{\prime} \subseteq S \text { such that } S^{\prime} \text { influential for } i\right\}
$$

and $S$ is a kernel if $F_{\mathbf{A}}(S) \neq \emptyset$ and $S^{\prime} \subset S$ implies $F_{\mathbf{A}}(S)=\emptyset$.

Definition 4. We say that the aggregation function $A_{i}$ of agent $i$ is exhaustive if $A_{i}\left(1_{S}\right)=$ 0 for some $S \subset N$ if and only if $A_{i}\left(1_{N \backslash S}\right)=1$.

It means that if agent $i$ does not take into account the opinion of $S$ (either 'yes' or 'no'), he should then follow the opinion of $N \backslash S$. Observe that in Example $5, A_{1}, A_{3}$ are not exhaustive. Examples of exhaustive and nonexhaustive functions will be given below.

The next result shows that the notion of influential coalition can fully describe terminal states.

Theorem 4. Suppose that all aggregation functions $A_{i}, i \in N$ are exhaustive. Take $S \subset N, S \neq \emptyset$. Then $S$ cannot be a terminal state if and only if, either there exist $S^{\prime} \subseteq N \backslash S, i \in S$ such that $S^{\prime}$ is influential for $i$, or there exist $S^{\prime} \subseteq S, i \notin S$ such that $S^{\prime}$ is influential for $i$.

(see proof in the appendix)

A similar result holds for Boolean terminal classes.

Theorem 5. Suppose that all aggregation functions $A_{i}, i \in N$ are exhaustive. Consider an interval $[S, S \cup K]$, with nonempty $S, K \subset N$, which forms a class. Then $[S, S \cup K]$ cannot be a Boolean terminal class if and only if, either there exists $S^{\prime} \subseteq N \backslash S, i \in S$ such that $S^{\prime}$ is influential for $i$, or there exists $S^{\prime} \subseteq S \cup K, i \notin S \cup K$ such that $S^{\prime}$ is influential for $i$.

(see proof in the appendix)

Unfortunately, it does not seem possible to find simple conditions for (general) regular classes with the help of influential coalitions.

Definition 5. An aggregation function $A_{i}$ is said to be decomposable if all its influential coalitions are singletons.

From Theorem 4, we can deduce the following.

Corollary 2. Suppose that all aggregation functions $A_{i}, i \in N$ are decomposable and exhaustive. Then there is no other terminal state than the trivial states if and only if the indirected influential graph $G_{A_{1}, \ldots, A_{n}}^{0}$ is connected. 
Proof. Since the $A_{i}$ 's are exhaustive, Theorem 4 ensures that the existence of terminal states depends solely on influential coalitions, which reduces to influential players by decomposability. But we have shown in the proof of Theorem 1 (iv) that ruling out all nontrivial terminal states by influential players is equivalent to have $G_{A_{1}, \ldots, A_{n}}^{0}$ connected.

Corollary 3. Suppose that all aggregation functions $A_{i}, i \in N$ are decomposable and exhaustive. Then there is no Boolean terminal class if and only if there is no subgraph $S$ of the influential graph $G_{A_{1}, \ldots, A_{n}}$ such that there is no arc ingoing to $S$, and there exists an agent $i \in N \backslash S$ which is not related to $S$.

Proof. As above, decomposability and exhaustiveness ensure that the existence of Boolean terminal classes depends only on influential players. Therefore, condition (B) (see the proof of Theorem 3 in the appendix) becomes necessary and sufficient (note that all Boolean classes are normal), which in turn is equivalent by Theorem 3 to the required conditions on the graph of influence.

\subsection{Some families of aggregation functions and their properties}

We exhibit now important families of aggregation functions (see Grabisch et al. (2009)).

Proposition 2. The family of generalized weighted means, defined by

$$
M_{f}\left(x_{1}, \ldots, x_{n}\right)=f^{-1}\left(\sum_{i=1}^{n} w_{i} f\left(x_{i}\right)\right), \quad\left(x_{1}, \ldots, x_{n}\right) \in[0,1]^{n}
$$

with $w_{i} \geq 0, i=1, \ldots, n, \sum_{i=1}^{n} w_{i}=1, f$ continuous automorphism on $[0,1]$, is a family of decomposable and exhaustive aggregation functions.

Proof. Suppose that $f(1)=1$ (the case $f(1)=0$ works the same), and that $S \subset N,|S|>$ 1 is influential. Then $M_{f}\left(1_{S}\right)=f^{-1}\left(\sum_{i \in S} w_{i}\right)>0$, which is equivalent to $\sum_{i \in S} w_{i}>0$. On the other hand, for any $i \in S$ we have $M_{f}\left(1_{i}\right)=f^{-1}\left(w_{i}\right)=0$, equivalent to $w_{i}=0$, a contradiction.

Suppose that there exists $S \subseteq N$ such that $M_{f}\left(1_{S}\right)=0$. By nonnegativity of the weights $w_{i}$, this is equivalent to $w_{i}=0$ for all $i \in S$. By the normalization condition this implies $\sum_{i \notin S} w_{i}=1$, hence $M_{f}\left(1_{N \backslash S}\right)=1$.

When $f=I d$, we recover the weighted arithmetic mean as in the DeGroot model. Note that $i$ is influential if and only if $w_{i}>0$.

The previous family is in some sense a family of quasi-linear aggregation functions. We introduce now a very different family since it is of the min-max type: the family of $k$-order statistics. The $k$ th-order statistic for some $k \in\{1, \ldots, n\}$ is $\operatorname{OS}_{k}(\mathbf{x})=x_{(k)}$, where $\mathbf{x} \in[0,1]^{n}$ and $x_{(1)} \leq x_{(2)} \leq \cdots \leq x_{(n)}$, i.e., $\operatorname{OS}_{k}(\mathbf{x})$ is the $k$ th smallest coordinate of $\mathbf{x}$. This family contains three important particular cases: $\mathrm{OS}_{1}$ is the minimum, $\mathrm{OS}_{n}$ is the maximum, and if $n$ is odd, $\operatorname{OS}_{\left\lfloor\frac{n}{2}\right\rfloor+1}$ is the median. Moreover, when restricted to 0-1 inputs as here, order statistics can model majority functions: the majority function with threshold $q$ for some $q \in\left\{\left\lfloor\frac{n}{2}\right\rfloor+1,\left\lfloor\frac{n}{2}\right\rfloor+2, \ldots, n\right\}$ is exactly $\mathrm{OS}_{n-q+1}$. 
$k$ th-order statistics are not exhaustive, for any $k=1, \ldots, n$, except in one case. Indeed, if $k \leq\left\lfloor\frac{n}{2}\right\rfloor$, we have $\mathrm{OS}_{k}\left(1_{S}\right)=\mathrm{OS}_{k}\left(1_{N \backslash S}\right)=0$ for $S \subseteq N$ such that $|S|=k$. If $k>\left\lfloor\frac{n}{2}\right\rfloor$, it suffices to take $S$ such that $|S|=\left\lfloor\frac{n}{2}\right\rfloor$. Then, $\operatorname{OS}_{k}\left(1_{S}\right)=0=\operatorname{OS}_{k}\left(1_{N \backslash S}\right)$, unless $n$ is odd and $k=\left\lfloor\frac{n}{2}\right\rfloor+1$. In the latter case, it can be checked that $\mathrm{OS}_{k}$ is exhaustive.

Suppose that agent $i$ has $\mathrm{OS}_{k}$ as aggregation function. As it can be easily checked, any set $S \subseteq N$ with $|S|=n-k+1$ is influential for $i$ if $k \leq\left\lfloor\frac{n}{2}\right\rfloor$, while for $k>\left\lfloor\frac{n}{2}\right\rfloor$, one must have $|S|=k$; only those sets are influential. In summary, we have shown:

Proposition 3. Consider $\mathrm{OS}_{k}$ for some $k \in\{1,2, \ldots, n\}$. Then:

(i) $\mathrm{OS}_{k}$ is not exhaustive, unless $n$ is odd and $k=\left\lfloor\frac{n}{2}\right\rfloor+1$, the latter case being the classical majority function with an odd number of agents (no tie). Consequently, min, max, median as well as other types of majority functions are not exhaustive.

(ii) If $A_{i}=\mathrm{OS}_{k}$, then the collection of influential sets for $i$ is $\{S \subseteq N$ s.t. $|S|=n-k+1\}$ when $k \leq\left\lfloor\frac{n}{2}\right\rfloor$, and $\{S \subseteq N$ s.t. $|S|=k\}$ when $k>\left\lfloor\frac{n}{2}\right\rfloor$. Consequently, no $\mathrm{OS}_{k}$ is decomposable, and the only influential set for min and max is $N$.

We briefly mention a third family of interest, namely the ordered weighted averages $(O W A)$, which are nothing else than convex combinations of order statistics:

$$
\operatorname{OWA}\left(x_{1}, \ldots, x_{n}\right)=\sum_{i=1}^{n} w_{i} x_{(i)}, \quad\left(x_{1}, \ldots, x_{n}\right) \in[0,1]^{n},
$$

with $w_{i} \geq 0, \sum_{i=1}^{n} w_{i}=1$. It is easy to see that these aggregation functions are not exhaustive since the order statistics are not. By contrast, they are decomposable if and only if all weights are positive. OWA functions are used to model "soft" majority, like "most of people say yes".

\section{Related literature on aggregation models}

The idea of the aggregation model where each agent aggregates the opinions of the others is not new, since it can be traced back at least to the model of DeGroot. We recall that in this model the opinion of agents is a number in $[0,1]$, and that the aggregation is done through a weighted arithmetic mean (convex combination). Also, the aggregated value is directly the opinion of the agent, which shows the fundamental difference with our model. In fact, our proposal is closer to the one of Asavathiratham (2000), although there are important differences in the dynamic aspect. In this model, the probability of an agent to say 'yes' is, as in the DeGroot model, a convex combination of the opinions of the other agents, where 'yes' is coded by 1, and 'no' by 0 .

We are not aware of other studies in the field of influence and social networks, using other means of aggregating opinions, nor considering a general class of aggregation function like in our model. It is however possible to find related studies in discrete mathematics concerning mainly Boolean functions, often applied to bioinformatics. There is a remarkable paper by Aracena et al. (2004), that we have already cited above. Here, functions from $\{0,1, \ldots, m-1\}^{n}$ to $\{0,1, \ldots, m-1\}^{n}$ are considered, which corresponds 
in our framework to $n$ agents having $m$ possible answers, and the aggregation gives directly the opinions of the agents after influence (hence the case $m=2$ is exactly our deterministic influence model). Aracena et al. study in particular the cyclic terminal classes, supposing some properties, like monotonicity of the aggregation functions and symmetry of the graph of influence. Rémy et al. (2008) provides also an interesting study of cyclic terminal classes when the aggregation functions are Boolean functions, with the restriction that transitions are of the type $S \rightarrow S \cup i$ or $S \rightarrow S \backslash i$, for $S \subseteq N$.

Lastly, we mention Mueller-Frank (2010) who provides a study of convergence applied to non-Bayesian learning in social networks. Here, aggregation functions which are continuous and a have a special property called "constricting" are considered. Supposing a graph depicting neighboring relations among agents, $A_{i}$ is constricting if $A_{i}(\mathbf{x})$ is comprised between the minimum and maximum of the $x_{j}, j \in N_{i} \cup i$, where $N_{i}$ is the set of neighbors of $i$. It is shown that for such functions, the process converges to a fixed point.

As it can be seen, all these studies consider particular cases of aggregation functions, and up to our knowledge there is no general study as the one we have undertaken in this paper.

\section{References}

J. Aracena, J. Demongeot, and E. Goles. On limit cycles of monotone functions with symmetric connection graph. Theoretical Computer Science, 322:237-244, 2004.

C. Asavathiratham. Influence model: a tractable representation of networked Markov chains. PhD thesis, Massachusetts Institute of Technology, Cambridge, MA, 2000.

V. Bala and S. Goyal. Conformism and diversity under social learning. Economic Theory, 17:101-120, 2001.

V. Bala and S. Goyal. Learning from neighbours. The Review of Economic Studies, 65(3): 595-621, 1998.

A. V. Banerjee. A simple model of herd behavior. Quarterly Journal of Economics, 107(3):797-817, 1992.

A. V. Banerjee and D. Fudenberg. Word-of-mouth learning. Games and Economic Behavior, 46:1-22, 2004.

R. L. Berger. A necessary and sufficient condition for reaching a consensus using DeGroot's method. Journal of the American Statistical Association, 76:415-419, 1981.

S. Broadbent and J. Hammersley. Percolation processes I. Crystals and mazes. Proceedings of the Cambridge Philosophical Society, 53:629-641, 1957.

A. Calvó-Armengol and M. O. Jackson. Like father, like son: social network externalities and parent-child correlation in behavior. American Economic Journal: Microeconomics, 1:124-150, 2009. 
B. Celen and S. Kariv. Distinguishing informational cascades from herd behavior in the laboratory. American Economic Review, 94(3):484-497, 2004.

M. H. DeGroot. Reaching a consensus. Journal of the American Statistical Association, 69:118-121, 1974.

P. DeMarzo, D. Vayanos, and J. Zwiebel. Persuasion bias, social influence, and unidimensional opinions. Quarterly Journal of Economics, 118:909-968, 2003.

G. Ellison. Learning, local interaction, and coordination. Econometrica, 61:1047-1072, 1993.

G. Ellison and D. Fudenberg. Rules of thumb for social learning. Journal of Political Economy, 101(4):612-643, 1993.

G. Ellison and D. Fudenberg. Word-of-mouth communication and social learning. Journal of Political Economy, 111(1):93-125, 1995.

N. E. Friedkin and E. C. Johnsen. Social influence and opinions. Journal of Mathematical Sociology, 15:193-206, 1990.

N. E. Friedkin and E. C. Johnsen. Social positions in influence networks. Social Networks, 19:209-222, 1997.

D. Gale and S. Kariv. Bayesian learning in social networks. Games and Economic Behavior, 45(2):329-346, 2003.

B. Golub and M. O. Jackson. Naïve learning in social networks and the wisdom of crowds. American Economic Journal: Microeconomics, 2(1):112-149, 2010.

M. Grabisch and A. Rusinowska. A model of influence in a social network. Theory and Decision, 69(1):69-96, 2010a.

M. Grabisch and A. Rusinowska. Measuring influence in command games. Social Choice and Welfare, 33:177-209, 2009.

M. Grabisch and A. Rusinowska. Influence functions, followers and command games. Games and Economic Behavior, 72(1):123-138, 2011a.

M. Grabisch and A. Rusinowska. A model of influence with an ordered set of possible actions. Theory and Decision, 69(4):635-656, 2010 b.

M. Grabisch and A. Rusinowska. A model of influence with a continuum of actions. Journal of Mathematical Economics, 2011b. DOI: 10.1016/j.jmateco.2011.08.004, forthcoming.

M. Grabisch and A. Rusinowska. Different approaches to influence based on social networks and simple games. In A. van Deemen and A. Rusinowska, editors, Collective Decision Making: Views from Social Choice and Game Theory, Series Theory and Decision Library C, Volume 43, pages 185-209. Springer-Verlag Berlin Heidelberg, 2010c. 
M. Grabisch, J.-L. Marichal, R. Mesiar, and E. Pap. Aggregation Functions. Number 127 in Encyclopedia of Mathematics and its Applications. Cambridge University Press, 2009 .

C. Hoede and R. Bakker. A theory of decisional power. Journal of Mathematical Sociology, 8:309-322, 1982.

X. Hu and L. S. Shapley. On authority distributions in organizations: equilibrium. Games and Economic Behavior, 45:132-152, 2003a.

X. Hu and L. S. Shapley. On authority distributions in organizations: controls. Games and Economic Behavior, 45:153-170, 2003b.

E. Ising. Beitrag zur theorie des ferromagnetismus. Zeitschrift für Physik, 31:253-258, 1925.

M. O. Jackson. Social and Economic Networks. Princeton University Press, 2008.

R. L. Keeney and H. Raiffa. Decision with Multiple Objectives. Wiley, New York, 1976.

H. Kesten. Percolation Theory for Mathematicians. Birkhauser, 1982.

A. Kirman. Ants, rationality, and recruitment. Quarterly Journal of Economics, 108(1): 137-156, 1993.

A. Kirman, C. Oddou, and S. Weber. Stochastic communication and coalition formation. Econometrica, 54(1):129-138, 1986.

U. Krause. A discrete nonlinear and nonautonomous model of consensus formation. In S. Elaydi, G. Ladas, J. Popenda, and J. Rakowski, editors, Communications in Difference Equations. Amsterdam: Gordon and Breach, 2000.

W. Lenz. Beiträge zum verständnis der magnetischen eigenschaften in festen körpern. Physikalische Zeitschrift, 21:613-615, 1920.

D. López-Pintado. Diffusion in complex social networks. Games and Economic Behavior, 62:573-590, 2008.

D. López-Pintado. Influence networks. Working Paper Series, WP ECON 10.06, 2010.

D. López-Pintado and D. J. Watts. Social influence, binary decisions and collective dynamics. Rationality and Society, 20(4):399-443, 2008.

J. Lorenz. A stabilization theorem for dynamics of continuous opinions. Physica A, 355: 217-223, 2005.

M. Mueller-Frank. A fixed point convergence theorem in Euclidean spaces and its application to non-Bayesian learning in social networks. Technical report, University of Oxford, 2010. http://ssrn.com/abstract $=1690925$.

R. B. Potts. Some generalized order-disorder transformations. Mathematical Proceedings, 48(1):106-109, 1952. 
E. Rémy, P. Ruet, and D. Thieffry. Graphic requirements for multistability and attractive cycles in a Boolean dynamical framework. Technical report, Institut de Mathématiques de Luminy, 2008. Working paper.

A. Rusinowska. Different approaches to influence in social networks. Invited tutorial for the Third International Workshop on Computational Social Choice (COMSOC 2010), Düsseldorf, available at http://ccc.cs.uni-duesseldorf.de/COMSOC2010/slides/invited-rusinowska.pdf, 2010.

\section{Appendix - Proofs of theorems}

\section{Proof of Theorem 1}

We need first the following lemma.

Lemma 3. Consider the following rule $\mathcal{R}$ :

$j$ influential in $A_{i}$ rules out every coalition $S \in 2^{N}$ such that $S \ni i$ and $S \not \supset j$.

Then rule $\mathcal{R}$ rules out every coalition $S \in 2^{N}, S \neq \emptyset, N$ if and only if the graph of influence $G_{A_{1}, \ldots, A_{n}}$ is strongly connected.

Proof. Sufficiency: we suppose that $G_{A_{1}, \ldots, A_{n}}$ is strongly connected and we consider a nonempty set $S \subset N$. Take all arcs of $G_{A_{1}, \ldots, A_{n}}$ having their terminal endpoint in $S$, and call $I(S) \subseteq N$ the set of initial endpoints of these arcs. Suppose that $I(S) \neq S$. Then there is an $\operatorname{arc}(j, i)$ with $i \in S$ and $j \notin S$. Hence $S$ is ruled out by $\mathcal{R}$. Suppose on the contrary that $I(S)=S$. Then from nodes of $S$, it is not possible to attain nodes in $N \backslash S \neq \emptyset$, a contradiction with the connectivity hypothesis.

Necessity: $\forall i \in N$, there must be an arc $(j, i)$ for some $j \neq i$, otherwise $S=\{i\}$ is not ruled out by $\mathcal{R}$. Hence in $G_{A_{1}, \ldots, A_{n}}$, each node has a predecessor. Therefore, for each nonempty $S \subset N$, we define $I(S)$, the set of initial endpoints of the arcs whose terminal endpoints are in $S$, and $I(S) \neq \emptyset$.

Suppose there exists a set $S \subset N$ such that $S=I(S)$. Then $S$ is not ruled out by $\mathcal{R}$ since there is no arc $(j, i)$ with $i \in S$ and $j \notin S$. Consequently, no such $S$ exists. We claim that this implies that $G_{A_{1}, \ldots, A_{n}}$ is strongly connected. Indeed, suppose it is not true. Then there exist distinct nodes $i, j$ such that $R(i)$, the set of nodes which can reach $i$ (connected by a directed path) does not contain $j$, or $R(j) \not \supset i$. Then $R(i)$ (or $R(j)$ ) would satisfy $R(i) \neq N$ and $I(R(i))=R(i)$, a contradiction with the assumption.

\section{Proof of Theorem 1}

(i) Since $A_{i}(1, \ldots, 1)=1$ for every aggregation function, when $\mathbf{s}=\mathbf{1}_{N}$, we have $\mathbf{x}=$ $\left(A_{1}\left(1_{N}\right), \ldots, A_{n}\left(1_{N}\right)\right)=(1, \ldots, 1)$. Therefore the next state is $N$ with probability 1 , which proves that $N$ is a terminal state. Now from the property $A_{i}(0, \ldots, 0)=0$, $\forall i \in N$, we deduce similarly that $\emptyset$ is a terminal state. 
(ii) Take any $S \neq N, \emptyset$. It is a terminal state if and only if $b_{S, S}=1$ and consequently $b_{S, T}=0$ for all $T \neq S$. This is equivalent to $\left(A_{1}\left(1_{S}\right), \ldots, A_{n}\left(1_{S}\right)\right)=1_{S}$, i.e., $A_{i}\left(1_{S}\right)=1$ if $i \in S$ and 0 otherwise.

(iii) Clear from (ii).

(iv) Suppose that $j$ is influential in $A_{i}$. Then $A_{i}\left(1_{j}\right)>0$, and by monotonicity $A_{i}\left(1_{S}\right)>$ 0 for all $S \ni j$. By (ii), this rules out every $S$ such that $S \ni j$ and $S \not \supset i$. Similarly, $A_{i}\left(1_{S}\right) \leq A_{i}\left(1_{N \backslash j}\right)<1$ for all $S \not \supset j$. By (ii) again, this rules out any $S$ containing $i$ but not $j$. In summary, the following rule $\mathcal{R}^{\prime}$ holds:

$j$ influential in $A_{i}$ rules out any $S \in 2^{N}$ such that $[S \not \supset j$ and $S \ni i]$ or $[S \ni j$ and $S \not \supset i]$.

Consider $\bar{G}_{A_{1}, \ldots, A_{n}}$ the graph of influence with all arcs reversed. Then the rule $\mathcal{R}^{\prime}$ on $G_{A_{1}, \ldots, A_{n}}$ is equivalent to the rule $\mathcal{R}$ of Lemma 3 on $G_{A_{1}, \ldots, A_{n}} \cup \bar{G}_{A_{1}, \ldots, A_{n}}=G_{A_{1}, \ldots, A_{n}}^{0}$. Therefore, ruling out every nonempty $S \subset N$ by $\mathcal{R}^{\prime}$ is equivalent by Lemma 3 to have $G_{A_{1}, \ldots, A_{n}}^{0}$ connected.

\section{Proof of Theorem 2}

Throughout this section we use the convenient shorthand

$$
\left(1_{S}, x_{K}\right):=(0 \cdots 0 \underbrace{1 \cdots 1}_{S} \underbrace{x_{1} x_{2} \cdots x_{k}}_{K})
$$

for vectors in $[0,1]^{n}$. The following straightforward lemma is central for proving the result.

Lemma 4. From each set $S \in 2^{N}$, the number of possible transitions is of the form $2^{k}$, for some $k \in\{0,1, \ldots, n\}$, where $k$ is the number of components in $\mathbf{A}\left(1_{S}\right)$ different from 0 and 1. More precisely, if $\mathbf{A}\left(1_{S}\right)=\left(1_{T}, x_{K}\right)$ with $\left.x_{i} \in\right] 0,1[$ for all $i \in K$, then $S$ has a transition to any set in the Boolean lattice $[T, T \cup K]:=\left\{S^{\prime} \in 2^{N} \mid T \subseteq S^{\prime} \subseteq T \cup K\right\}$.

Proof of Theorem 2 Consider a terminal class $\mathcal{C}$, and $S \in \mathcal{C}$. Unless $\mathcal{C}$ is reduced to a single state, $S$ cannot be empty because from the empty set there is no other transition than to itself, and so $\mathcal{C}$ would not be a class. One and only one of the following cases can happen:

(i) $\mathbf{A}\left(1_{S}\right)=1_{S}$. Then $S$ is a terminal state, i.e., $\mathcal{C}=\{S\}$.

(ii) $\mathbf{A}\left(1_{S}\right)=1_{T}, T \neq S$. There is transition from $S$ to $T$ with certainty. If for all sets in $\mathcal{C}$ the transitions are certain, the only possibility is that $\mathcal{C}$ is a cycle $S \rightarrow T \rightarrow \cdots \rightarrow S$.

(iii) $\mathbf{A}\left(1_{S}\right)=\left(1_{T}, x_{K}\right)$, with $\left.x_{i} \in\right] 0,1[$ for any $i \in K,|K|=k$. From Lemma 4 , there are $2^{k}$ transitions, which form the Boolean lattice $[T, T \cup K]$. Then necessarily, $[T, T \cup K]$ is included in $\mathcal{C}$, for if some set $L \in[T, T \cup K]$ does not belong to $\mathcal{C}$, there would be a transition from $S$ to $L$, i.e., an arc outgoing from the class, contradicting that it is a terminal class. 
0 . The case of terminal states has been studied in Theorem 1 .

1. We study the second case (cycles), and put $\mathcal{C}=\left\{S_{1}, \ldots, S_{k}\right\}$. If the sequence $S_{1}, \ldots, S_{k}$ with $k \geq 2$ is a cycle, we must have:

$$
\begin{aligned}
\mathbf{A}\left(1_{S_{1}}\right) & =1_{S_{2}} \\
\mathbf{A}\left(1_{S_{2}}\right) & =1_{S_{3}} \\
\vdots & =\vdots \\
\mathbf{A}\left(1_{S_{k}}\right) & =1_{S_{1}} .
\end{aligned}
$$

Suppose that the vectors $1_{S_{1}}, \ldots, 1_{S_{k}}$ are incomparable (i.e., no relation of inclusion occurs among the $S_{i}$ 's). Then no condition due to the nondecreasingness of the $A_{i}$ 's applies, and therefore there is no contradiction among the above equations. Conversely, suppose there exist $S_{i}, S_{j}$ in the sequence such that $S_{i} \subseteq S_{j}$. By monotonicity of $A$ this implies that $S_{i+1}=A\left(S_{i}\right) \subseteq A\left(S_{j}\right)=S_{j+1}$, etc. This causes a contradiction since we finally arrive at $S_{i} \subset S_{j} \subseteq \cdots \subseteq S_{i}$ (letting $S_{k+1}:=S_{1}$, etc.). By Sperner's lemma, we know that the longest possible sequence of incomparable sets has length $\left(\begin{array}{c}n \\ \left\lfloor\frac{n}{2}\right\rfloor\end{array}\right)$, hence the bound on $k$.

2. We suppose that $\mathcal{C}$ is a terminal class, which is neither a cycle nor a singleton. Then there exists a set $S^{\prime} \in \mathcal{C}$ with several possible transitions, i.e., $\mathbf{A}\left(1_{S^{\prime}}\right)=\left(1_{S_{1}}, x_{K_{1}^{\prime}}\right)$, with $\left.x_{i} \in\right] 0,1\left[\right.$, for all $i \in K_{1}^{\prime}$. We have $S_{1} \neq \emptyset$, otherwise a transition to $\emptyset$ would be possible, i.e., $\mathcal{C}$ is not terminal. Similarly, $S_{1} \cup K_{1}^{\prime} \neq N$, otherwise a transition to $N$ would be possible. There may be several such $S^{\prime}$ with same $S_{1}$ but different $K_{1}^{\prime}$. Call $S$ one of them with the largest $K_{1}^{\prime}$, and put $\mathbf{A}\left(1_{S}\right)=\left(1_{S_{1}}, x_{K_{1}}\right),\left|K_{1}\right|=: k_{1}$. Then the Boolean lattice $\mathcal{C}_{1}:=\left[S_{1}, S_{1} \cup K_{1}\right]$ is the set of transitions from $S$ and is included in $\mathcal{C}$.

2.1. Suppose that for any $T \in \mathcal{C}_{1}$, we have $\mathbf{A}\left(1_{T}\right)=\left(1_{S_{1}}, x_{K_{1}}^{T}\right)$, with $x_{i}^{T} \in[0,1]$, $i \in K_{1}$. Then all transitions from a set of $\mathcal{C}_{1}$ remain in $\mathcal{C}_{1}$. It follows from the assumption on $\mathcal{C}$ that $\mathcal{C}_{1}$ is a terminal class, and therefore $\mathcal{C}=\mathcal{C}_{1}$. Note that monotonicity of the aggregation functions entails that for any $T, T^{\prime} \in \mathcal{C}_{1}$,

$$
T \subseteq T^{\prime} \Leftrightarrow x_{K_{1}}^{T} \leq x_{K_{1}}^{T^{\prime}}
$$

i.e., the vectors $\left(x_{K_{1}}^{T}\right)_{T \in \mathcal{C}_{1}}$ form a Boolean lattice isomorphic to $\left[S_{1}, S_{1} \cup K_{1}\right]$.

Note that the $x_{K_{1}}^{T}$ must be such that $\mathcal{C}_{1}$ is strongly connected. This is achieved, e.g., if $\left.x_{i}^{T} \in\right] 0,1[$.

2.2 Suppose on the contrary that $\mathcal{C} \neq \mathcal{C}_{1}$, implying that there is some set $S^{\prime} \in \mathcal{C}$ with a different transition, say $\mathbf{A}\left(1_{S}\right)=\left(1_{S_{2}}, x_{K_{2}^{\prime}}\right)$, with $S_{2} \nsupseteq S_{1}$ or $S_{2} \cup K_{2}^{\prime} \nsubseteq S_{1} \cup K_{1}$. Again, among all $S^{\prime}$ with same $S_{2}$ but different $K_{2}^{\prime}$, choose $S$ with largest $K_{2}^{\prime}$ and put $\mathbf{A}\left(1_{S}\right)=\left(1_{S_{2}}, x_{K_{2}}\right),\left|K_{2}\right|=: k_{2}$. Then the Boolean lattice $\mathcal{C}_{2}:=\left[S_{2}, S_{2} \cup K_{2}\right]$ is the set of transitions from $S$ and is included in $\mathcal{C}$.

Examining the transitions of all sets in $\mathcal{C}$ we eventually conclude that $\mathcal{C}=\mathcal{C}_{1} \cup \mathcal{C}_{2} \cup$ $\cdots \cup \mathcal{C}_{p}$, where each $\mathcal{C}_{j}$ is a Boolean lattice $\left[S_{j}, S_{j} \cup K_{j}\right]$, defined as above. Note that at least one $K_{j}$ must be nonempty, otherwise each set has only one transition, and then the only way to have a class is to have a cycle, a case which is excluded here.

There are two cases concerning transitions among the subcollections $\mathcal{C}_{j}$. Let us take w.l.o.g. $\mathcal{C}_{1}$ as starting point. 
2.2.1. Suppose that for all $T \in \mathcal{C}_{1}$ we have $\mathbf{A}\left(1_{T}\right)=\left(1_{S_{2}}, x_{K_{2}}^{T}\right)$, with $x_{i}^{T} \in[0,1]$, $i \in K_{2}$, i.e., from any set of $\mathcal{C}_{1}$ there are only transitions to members of $\mathcal{C}_{2}$. Note that in this case there is no special relation between $S_{1}$ and $S_{2}$.

2.2.2. Suppose on the contrary that in $\mathcal{C}_{1}$ there is a set $S$ with transition to, say, $\mathcal{C}_{2}$, and another one $T$ with transition to $\mathcal{C}_{3}$. Then monotonicity relations among the sets in $\mathcal{C}_{1}$ induce monotonicity relations between $S_{2}, S_{3}$ and $S_{2} \cup K_{2}, S_{3} \cup K_{3}$.

If $S \subset T$, then monotonicity of the aggregation functions entails $\mathbf{A}\left(1_{S}\right) \leq \mathbf{A}\left(1_{T}\right)$. Supposing $\left.\mathbf{A}\left(1_{S}\right)=\left(1_{S_{2}}, x_{K_{2}}^{S}\right), x_{i}^{S} \in\right] 0,1\left[\right.$ for all $i$ in $K_{2}$ and similarly for $T$, this implies $S_{2} \subseteq S_{3}, S_{2} \cup K_{2} \subseteq S_{3} \cup K_{3}$ and $x_{i}^{S} \leq x_{i}^{T}$ for all $i$ in $K_{2} \cap K_{3}$.

If $S$ and $T$ are incomparable, then $S_{2}$ and $S_{3}$ can be also incomparable. In this case, for $S \cap T$ and $S \cup T$ the transitions must satisfy $\mathbf{A}\left(1_{S \cap T}\right) \leq\left(1_{S_{2} \cap S_{3}}, x_{K_{2} \cap K_{3}}^{S \cap T}\right)$, and similarly for $\mathbf{A}\left(1_{S \cup T}\right)$. Note that necessarily $S_{2} \cap S_{3} \neq \emptyset$ and $S_{2} \cup K_{2} \cup S_{3} \cup K_{3} \neq N$, otherwise the class would not be terminal. Also, from $\mathcal{C}_{1}$ there will be transitions to 4 different subcollections, with bottom elements $S_{2}, S_{3}$, a subset of $S_{2} \cap S_{3}$ (say, $S_{2 \wedge 3}$ ) and a superset of $S_{2} \cup S_{3}$, say $S_{2 \vee 3}$, respectively. Observe that the union of these subcollections has a least and a greatest element, which are $S_{2 \wedge 3}$ and $S_{2 \vee 3}$, respectively.

\section{Proof of Theorem 3}

The next lemma is fundamental.

Lemma 5. Suppose that $\mathcal{C}$ is a regular terminal class, with lower and upper bounds $S_{*}, S^{*}$. Then $A_{i}$ cannot have an influential player $j \in N \backslash S_{*}$ whenever $i \in S_{*}$, or an influential player $j \in S^{*}$ whenever $i \in N \backslash S^{*}$.

Proof. Suppose that $\mathcal{C}$ is a regular terminal class. Then for all $S^{\prime} \in \mathcal{C}$, we have

$$
A_{i}\left(1_{S^{\prime}}\right)=\left\{\begin{array}{lll}
1, & \text { if } i \in S_{*} & (*) \\
0, & \text { if } i \notin S^{*} & (* *)
\end{array}\right.
$$

Suppose $i \in S_{*}$ and $A_{i}$ has an influential player $j \in N \backslash S_{*}$. Take $S^{\prime} \in \mathcal{C}$ such that $j \notin S^{\prime}$. This is always possible since necessarily one of the $S_{k}$ 's does not contain $j$ (for if $j \in S_{k}$ for all $k=1, \ldots, p$, then $\left.j \in S_{*}\right)$. This implies $1>A_{i}\left(1_{N \backslash j}\right) \geq A_{i}\left(1_{S^{\prime}}\right)$, which contradicts $(*)$.

Suppose now that $i \notin S^{*}$ and $A_{i}$ has an influential player $j \in S^{*}$. Take $S^{\prime} \in \mathcal{C}$ such that $j \in S^{\prime}$. Again this is always possible since $j \in S^{*}$ implies $j \in S_{k} \cup K_{k}$ for some $k$. Then $A_{i}\left(1_{S^{\prime}}\right) \geq A_{i}\left(1_{j}\right)>0$, which contradicts $\left({ }^{* *}\right)$.

By applying the above lemma to every pair $S_{*}, S^{*}$ with $S_{*} \neq \emptyset, S^{*} \neq N$, one can obtain a sufficient condition (B) for forbidding any normal regular terminal class not reduced to the trivial terminal classes.

Condition (B): for any $\emptyset \neq S \subset N$, any $S \cup K \neq N, K \neq \emptyset$, there exist $i \in S, j \notin S$ or $i \notin S \cup K, j \in S \cup K$ such that $j$ is influential in $A_{i}$.

We prove that condition (B) is equivalent to the conditions of Theorem 3 . 
Proof of Theorem 3 We consider the following rule $\mathcal{R}^{\prime \prime}$, which is in fact Lemma 5:

$j$ influential in $A_{i}$ rules out every regular class with lower and upper bounds $S_{*}, S^{*}$, such that $i \in S_{*}, j \notin S_{*}$ (arc going into $S_{*}$ ) or $i \notin S^{*}, j \in S^{*}$ (arc outgoing from $S^{*}$ ).

We prove that rule $\mathcal{R}^{\prime \prime}$ rules out all normal regular classes with lower and upper bounds $S_{*}, S^{*}$ if and only if there is no subgraph $S$ of $G_{A_{1}, \ldots, A_{n}}$ without ingoing arcs, for which there exists $i \in N \backslash S$ such that no path from a node in $S$ to $i$ exists.

Necessity: Suppose such an $S$ and $i$ exist, and consider $R(i)$, the set of nodes that can reach $i$ by a path. Note that $R(i) \cap S=\emptyset$. Then a regular terminal class with lower and upper bounds $S, N \backslash R(i)$ is possible, because $S$ is not ruled out (no ingoing arrow), and $N \backslash R(i)$ can be ruled out only by an arc going into $R(i)$, which does not exist by definition of $R(i)$.

Sufficiency: take any normal regular class with bounds $S_{*}, S^{*}$, i.e., with $1 \leq\left|S_{*}\right|<$ $n-1$ and $2 \leq\left|S^{*}\right|<n$. If $S_{*}$ has an ingoing arc, it is ruled out by $\mathcal{R}^{\prime \prime}$. If it has no ingoing arc, then by hypothesis every node outside $S^{*}$ is linked to $S_{*}$ by a path. Since $N \backslash S^{*}$ is always nonempty, taking any node $i$ in $N \backslash S^{*}$, there is a path from $S_{*}$ to $i$, hence necessarily an arc outgoing from $S^{*}$. Therefore, $S^{*}$ is ruled out by $\mathcal{R}^{\prime \prime}$.

\section{Proof of Theorem 4}

Sufficiency: Take $S^{\prime} \subseteq N \backslash S, i \in S$, and suppose that $S^{\prime}$ is influential in $A_{i}$. Then $A_{i}\left(1_{S}\right) \leq A_{i}\left(1_{N \backslash S}\right)<1$, hence $S$ is not a terminal class by Theorem 1 (ii). Now, suppose that $S^{\prime} \subseteq S, i \notin S, S^{\prime}$ influential in $A_{i}$. Then $0<A_{i}\left(1_{S^{\prime}}\right) \leq A_{i}\left(1_{S}\right)$, which implies that $S$ is not a terminal class.

Necessity: Suppose that $S$ is not a terminal state. Then by Theorem 1 (ii), either there is some $i \in S$ such that $A_{i}\left(1_{S}\right)<1$ or some $i \notin S$ such that $A_{i}\left(1_{S}\right)>0$.

Suppose that for some $i \in S, A_{i}\left(1_{S}\right)<1$. Take the largest $S^{\prime} \supseteq S$ such that $A_{i}\left(1_{S^{\prime}}\right)<1$. We claim that $N \backslash S^{\prime}$ is influential for $i$. Indeed, we have $A_{i}\left(1_{S^{\prime}}\right)<1$. Also, $A_{i}\left(1_{N \backslash S^{\prime}}\right)=0$ would imply by exhaustivity of $A_{i}$ that $A_{i}\left(1_{S^{\prime}}\right)=1$, a contradiction with the definition of $S^{\prime}$. Therefore, $A_{i}\left(1_{N \backslash S^{\prime}}\right)>0$. Finally, we have for all $S^{\prime \prime} \supset S^{\prime}$, $A_{i}\left(1_{S^{\prime \prime}}\right)=1$, and the claim is proved.

Suppose now that for some $i \notin S, A_{i}\left(1_{S}\right)>0$. Take the smallest $S^{\prime} \subseteq S$ such that $A_{i}\left(1_{S^{\prime}}\right)>0$. Then $S^{\prime}$ is influential for $i$. Indeed, $A_{i}\left(1_{S^{\prime}}\right)>0$, and by exhaustivity of $A_{i}$ we must have $A_{i}\left(1_{N \backslash S^{\prime}}\right)<1$. Finally, for every $S^{\prime \prime} \subset S^{\prime}, A_{i}\left(1_{S^{\prime \prime}}\right)=0$ holds.

\section{Proof of Theorem 5}

Sufficiency: Take $S^{\prime} \subseteq N \backslash S, i \in S$, and suppose that $S^{\prime}$ is influential in $A_{i}$. Then $A_{i}\left(1_{S}\right) \leq A_{i}\left(1_{N \backslash S^{\prime}}\right)<1$, hence $[S, S \cup K]$ cannot be a Boolean terminal class, for any $K$. Now, suppose that $S^{\prime} \subseteq S \cup K, i \notin S \cup K, S^{\prime}$ influential in $A_{i}$. Then $0<A_{i}\left(1_{S^{\prime}}\right) \leq$ $A_{i}\left(1_{S \cup K}\right)$, which implies that $[S, S \cup K]$ is not a terminal class.

Necessity: Suppose that the class $[S, S \cup K]$ is not terminal. Then there exists $S^{\prime} \in$ $[S, S \cup K]$ such that either $A_{i}\left(1_{S^{\prime}}\right)<1$ for some $i \in S$, or $A_{i}\left(1_{S^{\prime}}\right)>0$ for some $i \notin S \cup K$. Therefore, we can proceed exactly like in the proof of Theorem 4. 